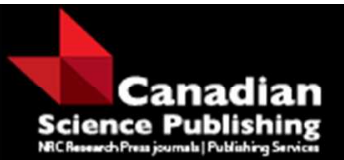

Canadian Journal of Forest Research

Revue canadienne de recherche forestière

More than Moran: Coupling statistical and simulation models to understand how defoliation spread and weather variation drive insect outbreak dynamics

\begin{tabular}{|r|l|}
\hline Journal: & Canadian Journal of Forest Research \\
\hline Manuscript ID & cjfr-2016-0396.R3 \\
\hline Manuscript Type: & Article \\
\hline Date Submitted by the Author: & $10-$ Mar-2017 \\
\hline Complete List of Authors: & $\begin{array}{l}\text { Nenzen, Hedvig; Université du Québec à Montréal (UQÀM), Biologie } \\
\text { Peres-Neto, Pedro; Université du Québec à Montréal (UQÄM), Biologie } \\
\text { Gravel, Dominique ; Universite de Sherbrooke, Biologie }\end{array}$ \\
\hline Keyword: & landscape ecology, model, outbreaks, climate autocorrelation, dispersal \\
\hline $\begin{array}{r}\text { Please Select from this Special } \\
\text { Issues list if applicable: }\end{array}$ & IUFRO 2016 Special Issue \\
\hline \multicolumn{2}{|l}{} \\
\hline
\end{tabular}


Title: More than Moran: Coupling statistical and simulation models to understand how defoli2 ation spread and weather variation drive insect outbreak dynamics

3

$4 \quad$ Authors: Hedvig K Nenzén ${ }^{1,2, *}$, Pedro Peres-Neto ${ }^{1}$, Dominique Gravel ${ }^{2}$

5

$6 \quad$ 1: Canada Research Chair in Spatial Modelling and Biodiversity, Département des sciences bi7 ologiques, Université du Quebec à Montréal, CP 8888, Succ. Centre Ville, Montréal, Quebec, H3C 8 3P8, Canada.* peres-neto.pedro@uqam.ca

9

2: Canada Research Chair on Terrestrial Ecosystems. Département de biologie, chimie et géographique, Université du Quebec à Rimouski, 300 Allée des Ursulines, Rimouski, Quebec, G5L 3A1, ${ }_{12}$ Canada ** dominique.gravel@usherbrooke.ca

$14 \quad *$ Corresponding author. hedvig.nenzen@gmail.com. Tel: 1-514-4987-3000 ext. 5239. Fax 1-514$15 \quad 987-4647$.

16 17 Current Addresses: * Department of Biology, Concordia University, Loyola Campus, 7141 Sher18 20 Canada. 
Understanding the processes that underlie species fluctuations is crucial to the development of efficient management strategies for outbreaks of destructive forest pests. Yet, the role of biotic and abiotic factors as well as their interactions in synchronizing outbreaks is not understood, despite many empirical and theoretical studies of species fluctuations. Here, we use a combined statistical-simulation model to investigate how defoliation spread and autocorrelated weather affect outbreaks of a major defoliator of North American boreal forest, the spruce budworm. We modelled the regional dynamics of spruce budworm and based the model on data collected from spatiotemporal aerial surveys of defoliation from 1968-2015 in Quebec, Canada. The effects of weather on local forest stand defoliation and mortality transitions, along with defoliation spread probability and distance, were estimated statistically. Simulations were run with these estimates to identify the effects of spatiotemporal weather autocorrelation on synchronicity of outbreaks. Defoliation spread together with weather variables was found to best fit the observed outbreak size. Simulation models suggest that positive temporal autocorrelation in weather promotes outbreaks, indicating that a series of suitable years could encourage outbreaks.

Keywords: landscape ecology, model, outbreaks, environmental autocorrelation, defoliation spread 

is an ongoing ecological challenge (Liebhold et al., 2004). Outbreaks are high population densities of

defoliating insects covering large areas and causing extensive tree mortality, making this phenomena of great concern for management decisions (Boyd et al., 2013). For example, spruce budworm (Choristoneura fumiferana) outbreaks profoundly structure the dynamics of North American boreal forests (Fleming, 2000; Pureswaran et al., 2016). Defoliation is so synchronized that during the last outbreak, 50 million hectares of Canada's forests were affected at the same time (Sturtevant et al., 2015), destroying 200 million $\mathrm{m}^{3}$ of wood and causing large economic and ecological impacts in the region (Morin et al., 2007). The multiple mechanisms hypothesized to synchronize budworm outbreaks are often studied separately, so their relative roles in driving outbreaks are not well understood which could limit efficient management actions. Here, we present a novel method to couple statistical and simulation models to tease apart the drivers of synchronous outbreaks of forest defoliating insects.

Hypotheses about what causes synchronous outbreaks are multiple and connected, but can be broadly divided into abiotic (i.e. environment) or intrinsic (i.e. defoliation spread) drivers (Liebhold et al., 2004). According to the abiotic hypothesis, spatially autocorrelated environmental variation affecting population dynamics synchronizes the abundance of isolated populations (the Moran effect, Moran, 1953). Alternatively, the intrinsic hypothesis suggests that synchrony occurs when individuals from a single population disperse rapidly throughout the landscape (Williams and Liebhold, 2000). In nature, multiple hypotheses may interact to produce outbreaks (Royama, 1984; Cooke et al., 2007). Previous empirical studies concentrated on isolated local populations (Tian et al., 2011), thus not considering the effect of possible dispersal from adjacent populations in driving population changes. Similarly, theoretical models may have underestimated the effects of dispersal as they also only used local population density data (Kendall et al., 1999). Such local population-level ap- 
63

64

proaches provided only a partial understanding of the mechanisms underlying outbreaks that can be driven by both regional environmental fluctuations and dispersal (Gilbert and O'Connor, 2013). As such, it is necessary to use landscape-level analysis of species dynamics to investigate the effects of both environmental autocorrelation and dispersal.

In order to represent the dynamics of insect outbreaks on a landscape-level, an alternative approach to population-level models, is to consider their population state as in either low (endemic) or high (epidemic) abundance. During endemic periods, budworm could be considered as 'absent' as they cause no detectable forest defoliation (Régnière and Nealis, 2007). During epidemic periods budworm densities are several orders of magnitude higher (Régnière and Nealis, 2007) and the could be considered as 'present' because they cause visible forest defoliation. Therefore, instead of modelling the presence of defoliators we model the presence of defoliation. Large-scale dynamics thus emerge from the local defoliation (from absent to present) and mortality (from present to absent) transitions. Fortunately, large-scale, multi-year empirical defoliation data (e.g., aerial surveys) is readily available, yet rarely used to identify parameters and simulate models (Zhou and Liebhold, 1995). We use defoliation data as a proxy for high insect densities. Defoliating insect species such as the budworm show extreme density fluctuations, which leads us to propose that their dynamics can be sufficiently expressed by presences/absences instead of abundances.

The spatial dynamics of defoliation easily map onto metapopulation and epidemiological models. Metapopulation theory (Levins, 1969) represents transitions between empty and occupied patches driven by colonization and extinction. Similarly, epidemiological models (Kermack and McKendrick, 1927; Keeling and Rohani, 2008) track either negligible or disease-causing densities of the infectious agent among individual hosts. The two methods are mathematically equivalent in some instances (Grenfell and Harwood, 1997) and are supported by extensive theoretical development (Hanski, 1998). For example, spatially-explicit vaccination of the most vulnerable locations can reduce the 
87 size of epidemics of infectious diseases (Keeling et al., 2003). Insights from epidemiological models

could be used to develop more effective spatial management strategies of insect outbreaks.

Transition from endemic to epidemic states is a binomial stochastic process whose drivers can be evaluated from empirical data. Stochastic cell occupancy, state-and-transition, and occupancy models, are all essentially parameterized metapopulation models of species dynamics in space (Moilanen, 2004; Buckland and Elston, 1993; Bestelmeyer et al., 2004). Statistical models have been used to determine how local stand transitions of various insect species depend on environment and potential defoliation spread from already defoliated neighboring cells (Bouchard and Auger, 2014; Kärvemo et al., 2016). The transitions observed in aerial surveys could be used to parameterize a model of insect defoliations according to simple rules of defoliation spread and environmental effects. According to metapopulation and epidemiological theory, the transition from endemic to epidemic is expected to be conditional on both defoliation spread and abiotic conditions. Here, we propose that such an approach of insect outbreaks can be used to investigate the effects of intrinsic and extrinsic drivers of outbreaks.

The environment is usually autocorrelated in space and time (Koenig, 2002). Positive autocorrelation, when environmental values are more similar among adjacent temporal or spatial units than expected by chance, may affect species fluctuations and defoliation spread (Ruokolainen et al., 2009). Synchronous outbreaks may be produced by suitable environments in many locations (Moran effect, spatial autocorrelation). Recent theoretical and experimental studies suggest that suitable environmental conditions over consecutive periods further synchronize populations (temporal autocorrelation, Gonzalez and Holt, 2002; Massie et al., 2015), but this mechanism has never been demonstrated in nature. We propose that a simulation model parameterized from empirical transitions can assist in disentangling the relative importance and the interactions between drivers of outbreak dynamics.

The goal of our study is threefold: 1) determine how weather and defoliation spread drive ob- 
served budworm outbreak and mortality transitions; 2) quantify the relative importance of these two variables; and 3) determine if spatial or temporal weather autocorrelation synchronize outbreaks. We parameterized a lattice-based metapopulation model from observed outbreaks in Quebec, Canada. We predict that if the model reproduces the observed outbreaks using the observed autocorrelated weather variables, it suggests that the Moran effect drives budworm outbreaks. Alternatively, if the model reproduces the observed outbreaks with non auto-correlated weather, then it would suggest that defoliation spread is the most important driver. As this study only considers defoliation spread and weather, we cannot deduce whether other hypotheses not considered here drive outbreaks. We used budworm as an example species but our coupled statistical-simulation metapopulation model could be applicable to any species that displays large-scale synchrony.

\section{Materials and methods}

\section{Coupled statistical and simulation model}

We considered a metapopulation approach in which a single spatial unit is a cell, and multiple cells together constitute a landscape (Fig. 1). Each cell can be in two different states: forest $F$ with endemic budworm densities that do not cause defoliation, or defoliated forest $I$ with epidemic budworm densities that show signs of defoliation (or infection, hence $I$ ). As such, these two cell states correspond to observed endemic and epidemic population densities that vary over orders of magnitude (Cappuccino et al., 1998). We studied the transition from forest to defoliation (defoliation transition $F \rightarrow I$ or $F \rightarrow I$, colonization) and the transition from defoliation back to forest (mortality transition $I \rightarrow F$, extinction, Fig. 2). Stochastic state transitions $(F \rightarrow I$ and $I \rightarrow F)$ across all cells (landscape) generate a fluctuating proportion of $F$ and $I$ cells over time.

Our model has two parts. We start by statistically estimating the transition probabilities of defo- 
liation and mortality given defoliation spread constraints and climatic conditions, and then we use the transition probabilities as parameters in a simulation model to test the influence of autocorrelation on outbreaks. Defoliation of a single forest cell resulted from two processes corresponding to the following two hypotheses, respectively: 1) by increasing the insect population density due to favourable weather; 2) by dispersing individuals arriving from neighboring defoliated cells (Fig. 2 arrows). A candidate set of statistical submodels were chosen a priori based on defoliation spread and weather hypotheses to assess the relative contribution of these two processes (i.e., weather and defoliation spread) in driving large-scale outbreaks. The probability of defoliation in cell $i$ was modeled as a log-linear model of weather and defoliation spread:

$$
\text { Probability }\{F \rightarrow I\}_{i}=\beta_{0}+\beta_{n} E_{i}+\alpha_{n} K_{i}
$$

where $\beta_{0}$ is the intercept, $E_{i}$ is the set of weather variable, $\beta_{n}$ is the set of parameters representing the effect of weather variables on the transition probability, $K_{i}$ is the proportion of defoliated neighbors around cell $i$ and $\alpha_{n}$ is the parameter representing this effect. We tested up to third degree polynomial, i.e. $n$ up to 3 (eqn 1), for both weather and defoliation spread variables. This accounted for complex responses while obtaining parametric relationships for the simulation model. The transition probability of mortality from defoliation to forest is only dependent on weather. The mortality transition is set by parameter $\gamma$ :

$$
\text { Probability }\{I \rightarrow F\}_{i}=\gamma E_{i}
$$

First, we performed model comparison to select the best-fitting statistical model representing the observed transitions. Second, the statistical model of transition probabilities was used to carry out simulation 'experiments' to investigate the effect of spatiotemporal weather autocorrelation on outbreak dynamics. Our statistical models can only describe the effect of weather for single time 
153

154

steps and cells, whereas simulations integrate dynamics across the entire landscape and over time. We measured the total proportion of defoliated cells in the landscape through time generated by the simulation model and assessed if they were similar to observed outbreaks. We tested the influence of weather autocorrelation on outbreaks by randomizing weather variables $E_{i}$, which maintained climatic values but removed autocorrelation structure.

\section{Spatiotemporal data}

\section{Spruce budworm defoliation distribution}

The Ministère des Forêts, Faune et Parc du Quebec (MFFP, 2014) have tracked the temporal (annual) and spatial distribution of budworm outbreaks based on aerial surveys and recorded mapped polygons indicating areas of defoliation in Quebec province, Eastern Canada. The data span 1968 through 2015, which include one full outbreak (1968-1995), along with the beginning of an ongoing outbreak which started around 2010 (Fig. 1).

We converted these polygons to a lattice of $10 \mathrm{~km} \times 10 \mathrm{~km}$ geographical cells (patches, forest stands, from here on referred to as cells arranged in a continuous lattice, Fig. 1), which formed the basis for our coupled statistical-simulation model. Because recent polygons were mapped to a smaller scale than older data, data across all years were aggregated to the same cell size. We overlaid $1 \mathrm{~km} x$ $1 \mathrm{~km}$ spaced points on the original defoliated polygons, and then resampled this to a regular grid of $10 \mathrm{~km} \times 10 \mathrm{~km}$ cells so if at least one of the points within the cell was defoliated, the cell was classified as defoliated (following Bouchard and Auger, 2014). This aggregation also minimized small-scale landscape variations. Any level of defoliation (light, moderate or serious defoliation) within polygons were registered as $I$. We assumed that non-defoliated areas were $F$ and that mortality occurred when defoliation was no longer observed, even though it is unknown if the forest dies or survives defoliation. In total, the study area had 6446 cells after removing cells that were never defoliated. 
Each cell was mapped for 46 years, yielding 283272 transition events (Tables ??, ??).

After mortality from defoliation, forest regrowth usually takes around 40 years (Burns and Honkala, 1990), but occasionally a cell was observed to become re-defoliated before full regrowth. These fast transitions may have arisen because of detection errors and as such were not considered here as representative of the naturally observed defoliation and mortality processes we aim to model. To account for this issue, if a cell became defoliated, not defoliated and then re-defoliated within five years, we converted the intermediate years to the defoliated state.

\section{Weather variables}

Historical weather variables covering the same spatiotemporal extent as the defoliation data were obtained from publicly available data. In this data set, weather station data were interpolated to continuous rasters using the ANUSPLIN method (McKenney et al., 2011). Multiple weather variables, in particular extreme temperatures, influence outbreak severity and occurrence (Candau and Fleming, 2005). We consequently selected eight potential annual and seasonal weather variables (and elevation, Table 1) for the analysis. Weather one, two and three years before each year $t\left(E_{t-1}, E_{t-2}\right.$ and $\left.E_{t-3}\right)$ were also included as weather variables may have a delayed effect on insect population growth and mortality (Aukema et al., 2008), and because there is a lag between population growth and crossing the endemic-epidemic threshold where defoliation is detectable. All weather variables were standardized within the range $0-1$ (max-obs)/(max-min) to facilitate comparison between parameters.

\section{Defoliation spread variables}

The expansion of budworm defoliation to an endemic-density cell is more likely to occur if there are defoliated cells in the vicinity (Bouchard and Auger, 2014). Note that defoliation spread indirectly represented the successful dispersal of insect individuals. We considered defoliation spread 
kernels $K_{i}$ to represent propagule pressure around the neighbourhood of cell $i$. We compared $K_{i t-1}$ and $K_{i t-2}$ computed one and two years prior to the transition $t$ and $t+1$ given that egg-laying adults produced a population increase the following year when larvae emerge. We used two methods and a range of neighborhood sizes in each method to evaluate defoliation spread kernels that characterize defoliation spread:

The autologistic kernel represented the proportion of defoliated cells within a certain distance (Yackulic et al., 2015). All cells within the neighborhood were given equal weight. $K_{i}$ of each potential donor cell $i$ was the number of defoliated cells $j$ within distance $d . K_{i}$ is standardized by the total number of neighbors so that $K$ is one if all neighbours are defoliated. We calculated $K_{i}$ for $d=2-30$ cells in increments of 1 because the maximum radius of 30 cells covered the majority of the landscape (89 x 176 cells).

The negative exponential kernel included all cells in the landscape but gave a higher weight to cells close to the focal cell, allowing long-distance defoliation spread (i.e. beyond the threshold distance in the autologistic kernel). The negative exponential kernel is the sum of the weighted distances between all cells (Moilanen, 1999):

$$
K_{i}=\sum_{j \neq i} \exp \left(-\delta d_{i j}\right)
$$

where $\delta$ is the average defoliation spread distance and $d_{i j}$ is the distance in number of cells between cell $i$ and $j$ (in this equation $\alpha$ traditionally represents the effect of distance, but we use $\delta$ to avoid confusion with $\alpha$ probability of defoliation spread). We calculated defoliation spread variables varying $\delta=2-30$ in increments of 2 because this range captured extremes of both short and long-distance defoliation spread (Table ??). The values of negative exponential defoliation spread variable $K_{i}$ were standardized to $0-1$ (max-obs)/(max-min) to be on the same scale as the autologistic kernel. 


\section{Statistical models}

We determined how weather and defoliation spread influenced transition probabilities by tracking the state transitions of each cell in the observed defoliation data, and estimating how the probabilities were affected by weather and defoliation spread. We constructed separate statistical models for the defoliation $F \rightarrow I$ and mortality $I \rightarrow F$ state transition probabilities. Transition was modelled as a binomial process using logistic regression. The selected weather and defoliation spread variables described above were used as predictors of forest transitions (equation 1 - 2).

Models were evaluated in two ways: model fit (accuracy) and predictive performance on independent data. Goodness-of-fit metrics to assess model fit were $\triangle A I C$ and pseudo $r^{2}$ for logistic regressions, $r^{2}=1-($ residualdeviance/nulldeviance) . Cross-validation to assess predictive performance was performed on a random subset of the data and on the second outbreak. Random crossvalidation was performed by splitting the data in half, fitting the model on the subset and then estimating model performance on the remaining validation data. This was repeated 10 times and the average error calculated. Temporal cross-validation was performed by fitting the model to the first outbreak (1968-1992) and predicting the model on the second outbreak (1993-2015). We did not estimate temporal cross-validation in $I \rightarrow F$ transitions because there were little data from the second outbreak.

\section{Model comparison}

We tested which potential defoliation spread kernel $K_{i}$ and kernel parameters $d$ and $\delta$ best fitted the data. Given that the defoliation spread variables were highly correlated (first axis of a Principal Components Analysis on the correlation matrix explained $84 \%$ of the variation, Fig. ??), we used forward stepwise model selection to rank models with each defoliation spread kernel separately. Only the best-supported kernel was retained for all subsequent analyses (according to AIC, Figs. 
??).

The full regression model had one defoliation spread and all eight weather variables with three time delays as predictors (Table 2). We used the first and three best weather variables and interactions between them to construct submodels. To determine which candidate weather variable best explained the observed budworm defoliation and mortality transitions, we used backward stepwise model selection because weather variables were not collinear (the first PCA axis on the correlation matrix explained $50 \%$ of the variation, Figs. ??). We estimated a geographical null model to assess if outbreaks were independent of weather and directional in space (anisotropic). Latitude and longitude were used as predictors for the geographical null model. We also estimated a defoliation spread null model in which defoliation spread was not limited by distance (global defoliation spread). Proportion defoliated area in the previous year was the global defoliation spread predictor, and was compared to the best-fitting distance-based defoliation spread kernel. To assess the effect of host forest species we also tested the effect of dominant host species (boreal, deciduous or mixed) but this variable was not statistically important (Table ??).

\section{Simulation model}

We simulated outbreaks using the estimated defoliation and mortality transition probabilities from the full statistical model to investigate the effect of spatiotemporal autocorrelation of weather variables on the outbreak dynamics. We ran stochastic simulations with the statistical transition probabilities and the observed climatic fluctuations, so the simulations occurred in the same landscape and over the same years as the observed outbreaks. Uncertainty can propagate up and yield different large-scale dynamics even if the model successfully represents the local dynamics (here transitions, Peters et al., 2004).

Simulations were initialized with the 1970 weather and run for 45 years. Depending on the state 
of chosen cell $i(F$ or $I)$, transition probabilities Probability $\{F \rightarrow I\}_{i}$ and Probability $\{I \rightarrow F\}_{i}$ were applied (as explained above, equations 1 and 2). The defoliation spread kernel $K_{i}$ was calculated in each stand and year from the cells defoliated in the previous year. We then added weather and defoliation spread parameters exactly as in the statistical model to obtain spatiotemporal transition probabilities that ranged $0-1$ depending on stand weather and neighbourhood defoliation (equation 1). The final probabilities were then compared to uniformly distributed random values $0-1$ to determine if a transition occurs, so transitions were stochastic but influenced by the probabilities. We used asynchronous updating of the landscape by randomly picking and updating 6446 cells per time step in order to approximate continuous spatial dynamics (Durrett and Levin, 1994).

We carried out simulations with and without autocorrelated weather to understand if structured weather variation drives outbreaks (Figure 1, panels in Fig. 4). We randomized weather variables, thus maintaining the frequency distribution of values but removing the autocorrelation structure. First, we randomized weather conditions across time-steps, within each cell, to remove temporal autocorrelation. Then we randomized weather conditions between cells, within each time-step, to remove spatial autocorrelation. We randomized weather in both space and time to remove both temporal and spatial autocorrelation. Simulations with average weather values from all years and locations were carried out to test how weather variation affects outbreak dynamics. Finally, to investigate if the estimated defoliation spread kernel was important in producing outbreaks, we also implemented global defoliation spread with the original weather variables.

We characterized simulated outbreaks (30 repetitions per spatiotemporal autocorrelation) in time and space to compare them to observed outbreaks. Simulated defoliations were stochastic and so cannot be compared cell-by-cell to observed defoliations. Instead, to examine if simulated outbreaks were as large, i.e. as spatially synchronous as observed outbreaks, we recorded the proportion of the landscape in state $I$ per year. We also calculated the maximum, standard deviation, and temporal 
autocorrelation of proportion $I$. Real outbreaks are known to be spatially clustered (i.e. defoliated cells were often connected by their 8 closest neighbors when considering a rasterized landscape, Fig. $1 \mathrm{~b}$ ), and so we determined if the simulation model was able to reproduce this property. We calculated the maximum cluster size and number of outbreak clusters per simulation to compare the spatial characteristics of observed and simulated landscapes.

\section{Results}

Overall, the statistical model identified that budworm defoliation spread most often occurs over short distances. We found that the negative exponential kernel best fit the observed defoliation transitions (Fig. ??, Fig. 3 for example). The defoliation spread probabilities were generally below 0.5 , indicating that defoliation spread was episodic and did not always occur. We identified that lagged spring and summer temperatures best explained the defoliation and mortality transitions. The three strongest climatic drivers of the $F \rightarrow I$ transition were in order of importance: maximum summer temperature three years before, summer minimum temperature three years before and number of growing degree days 2 years before (Fig. ??, Table ??). Climatic transition probabilities were low, around 0.1 for the most suitable conditions (Fig. ??, ??). Minimum spring temperature three years before, precipitation three years before, and minimum temperature of the coldest period two years before were the strongest predictors of mortality transitions (Fig. ??, Table ??).

The model comparison indicated that both weather and defoliation spread drivers affected outbreaks, but their relative importance depended on how models were evaluated. Models with only weather variables were more accurate (according to AIC), while models with only defoliation spread predicted the second outbreak better (according to temporal cross-validation, Table 2, Table ??). The full weather model, i.e. with all climatic variables and third degree response, was the most accurate model when predicting on randomly selected data from both outbreaks (cross-validation according 
to AIC, Table 2). However, the full model was less successful at predicting the second outbreak (according to temporal cross-validation, Table 2). The model with the best predictive performance was a submodel with three weather variables, defoliation spread and interactions between them (according to temporal cross-validation, Table 2). Defoliation spread was the best unique variable in terms of both accuracy and predictive performance (according to AIC and cross-validation, Table 2). Adding interactions between weather and defoliation spread increased model fit, though it decreased predictive power (according to AIC and temporal cross-validation, Table 2). Models with interaction so closely fit to the first outbreak are not general enough to predict the second outbreak (overfit). The geographical null model did not fit outbreaks well, indicating that weather was important in synchronizing outbreaks.

Simulations with the parameterized metapopulation model revealed that successive periods of temporally autocorrelated weather synchronized outbreaks. Retaining only the temporal weather autocorrelation produced outbreaks that covered $70 \%$ of the landscape, compared to the $90 \%$ observed (example simulations Fig. 4, Fig. 5 a). Simulations with the original autocorrelated weather also produced outbreaks that were as large as the observed outbreaks (Fig. 5, a, b). When retaining only spatial autocorrelation (i.e., removing temporal autocorrelation), outbreaks did not occur during the same years as observed outbreaks and were on average smaller (40-60\%, Fig. 5 a), indicating that the model was less sensitive to spatial autocorrelation. Simulations with constant weather had $10 \%$ landscape defoliation throughout the simulations with no variability.

Temporal dynamics were in general well captured, and spatial dynamics less so. Randomizing weather in time produced outbreaks with lower next-year temporal autocorrelation (correlations $0.7-0.9$ with 1 year time lag, Fig. 5 c), with long-range positive temporal autocorrelation (6-16 years, Fig. 5 d). Even spatially randomized climatic conditions occasionally resulted in outbreaks that were as temporally autocorrelated as observed outbreaks. The difference between spatially au- 
tocorrelated (random time) and completely random weather conditions was small, again indicating that spatial autocorrelation has a weak effect on outbreak dynamics. Simulated outbreaks were more fragmented in space than observed ones. Simulated outbreak clusters were more numerous and smaller in size than the observed ones (Fig. 1,5e,f). Simulations with global defoliation spread and observed spatiotemporal autocorrelation yielded landscapes with high proportions of defoliated cells but reflected neither the temporal nor the spatial characteristics of observed outbreaks (Fig. 5).

\section{Discussion}

We performed a coupled statistical and simulation analysis of 48 years of spruce budworm outbreak observations to understand how defoliation spread and weather drive the dynamics. The estimated parameters from the best statistical model were incorporated into a simulation model to investigate the role of spatiotemporal autocorrelation on the initial development of outbreaks and their spatial structure. The same method can be applied to outbreaks of other insect species that are mapped by defoliation.

We found that defoliation spread between forest cells occurred occasionally over long distances, however short-distance defoliation spread was more common. Individual-based dispersal studies show that adult moths can efficiently travel $150 \mathrm{~km}$ (Sturtevant et al., 2013), and the maximum recorded distance is $450 \mathrm{~km}$ (Greenbank, 1980). The greater importance of the negative-exponential kernel over the autologistic version indicates that defoliation spread was essentially driven by strong short-distance defoliation spread events and occasional long-distance defoliation spread events. For instance, the probability of defoliation spread was low though a cell was surrounded by defoliated cells in a suitable weather (Fig. ??). The budworm population is genetically well-mixed during outbreaks (James et al., 2015), supporting effective long-distance dispersal. However, defoliation spread was still limited by distance as the null model with global defoliation spread badly fit the data (Table 
2). Metapopulation models are often estimated from static (snapshot) distributions and individual dispersal observations (Moilanen, 1999). In contrast to these static methods and due to the detailed spatiotemporal nature of our data, we characterized effective defoliation spread. The strength and shape of dispersal is often the unknown factor in predictive models, and thus in understanding outbreaks (Myers and Cory, 2013). Although dispersal may not be completely represented by defoliation data, our method describes an effective method to quantify defoliation spread directly from spatiotemporal occupancy data.

We attempted to disentangle the effects of weather and defoliation spread with an innovative coupling of statistical analysis and simulation modelling. In the end, our results indicate that both weather and defoliation spread interact to drive mortality. Previous studies may have over-estimated the role of the Moran effect in driving population fluctuations because they did not investigate spatial data and thus discounted dispersal. Purely temporal analyses pool data across large regions (Tian et al., 2011), thus possibly confounding local defoliation spread events with climatic effects. Spatial analyses suggest that weather drives dynamics if the spatial autocorrelation range of weather is longer than the spatial autocorrelation range of outbreaks (autocorrelation range distance measured in kilometres, Peltonen et al., 2002). Models that exclude dispersal cannot characterize how weather drives epidemic transition events separately from dispersal. Recent process-based statistical studies testing both weather and dispersal (intrinsic and extrinsic) hypotheses have shown that dispersal is more important than environment in synchronizing outbreaks (Preisler et al., 2012; Veran et al., 2014). Similarly, we found that defoliation spread was an important factor in synchronizing defoliations in space, although it required an autocorrelated weather trigger (simulations with constant weather vs. simulations with weather, Figure 4).

Among the abiotic drivers, a suite of weather predictors best described the observed transitions. We found that delayed spring and summer temperatures influenced both defoliation and mortality 
transitions, suggesting that weather during the reproductive season causes populations to reach epidemic insect densities years later, similar to what Aukema et al. (2008) observed. Multiple weather variables are necessary to reproduce outbreak dynamics because these may affect insect development periods and life histories differently. In general, winter temperatures affect overwintering survival, maximum spring temperature affects larval emergence, and summer temperatures affect feeding rates (Régnière et al., 2012; Gray, 2013). The full model with all weather variables was more accurate, suggesting that a specific combination of weather characteristics was important ('a perfect storm', Wilmers et al., 2007). Moreover, interactions between predictors increased fit, and may indicate that weather and defoliation spread interact (e.g., defoliation spread is greater in warmer temperatures).

The difficulty in identifying a single weather driver may be explained statistically. First, it is difficult to fit autocorrelated predictor variables (Kissling and Carl, 2008). Spatiotemporal autoregressive models that account for spatiotemporal autocorrelation are not generalized to logistic regression with complex response functions (Cressie and Wikle, 2011, but see Griffith and Peres-Neto, 2006). Moreover, our goal is not to remove autocorrelation, but to understand how it affects species dynamics. Cross-validation suggested that the full model overfit the data and included the maximum number of variables to produce a spurious relationship. Simpler models with fewer variables are generally preferred for prediction purposes (Cuddington et al., 2013).

The simulation model suggests that non-random weather variation produced outbreaks even though weather itself was a weak statistical predictor (as in the Moran effect). Simulation models can draw such conclusions because they simulate several years, not just one year ahead. The model reproduced the emergent temporal characteristics of budworm outbreaks; 0.9 temporal autocorrelation with a one year lag, and 10 year range in positive autocorrelation (Fig. 5 c, d). We identified that temporal weather autocorrelation drives large-scale outbreaks, because retaining only temporal 
autocorrelation produced outbreaks that were similar to both spatial and temporal outbreak characteristics. Non-random temporal environmental variation has been shown to inflate the Moran effect in microcosm experiments (Gonzalez and Holt, 2002; Massie et al., 2015) and theoretical population models (Vasseur, 2007). For the budworm, several consecutive dry summers have been associated with defoliations (Greenbank, 1956). This simulation model allowed us to identify the importance of autocorrelated temporal weather variation at the landscape scale. However, because weather has a high spatial autocorrelation (analysis not shown), randomizing weather in space does not change dynamics and may have led us to underestimate the role of spatial autocorrelation.

To estimate the effect of weather and defoliation spread, we used to most complete set of spatiotemporal data available for the spruce budworm, but this presence-absence dataset still presents some limitations. Only the data for Québec province is publicly available, so our study is bounded by areas with outbreaks that we did not track but which could have nevertheless affected outbreaks. For example, defoliation could have spread into Québec from the provinces of Ontario and New Brunswick. This edge-effect could have led us to underestimate the role of dispersal in our study. Further, aerial survey data may omit low-density population dynamics that are under the defoliation threshold. This cross-scale assumption is partially accounted for by the statistical significance of the weather variables three years previously to the defoliation transition. A large-scale study may find different climate-responses than a population-level study. For example, Robert et al. (2012) showed that tree-ring patterns indicate that forest management determined spruce budworm outbreaks. Finally, as we use observed data we cannot identify causes, only correlations between outbreaks and climate. However, a simulation model was employed to improve the plausibility of the results, and can be considered as the only type of 'experiment' possible to investigate landscape-scale outbreaks. Moreover, theoretical experiments such as ours can suggest future avenues for investigation.

Our simulation model showed that spatiotemporal autocorrelation affected outbreaks, but it did 
not completely reproduce the observed dynamics. Observed defoliation spread events are episodic in time and clustered in space, yielding a small number of large outbreak clusters (maximum 30 clusters and a single cluster covering $85 \%$ of the study area, Fig. 5 e, f). Defoliation spread is difficult to model because it is inherently stochastic and may be driven by unpredictable atmospheric conditions such as wind direction (Sturtevant et al., 2013). Because our model already contained all possible observed climatic spatial autocorrelation, it is possible that additional hypotheses are required to completely predict outbreak patterns (Liebhold et al., 2004). For instance, the Allee effect decreases mating success at endemic population densities (Régnière et al., 2013) and may alter dispersal and establishment, and possibly landscape-scale outbreak dynamics. Spatially correlated distributions of natural enemies such as parasitoids may synchronize insect densities (Roland and Taylor, 1997; Pureswaran et al., 2016), but there is little available empirical data. The effect of local environmental conditions such as forest composition may further mediate natural enemy interactions (Bouchard and Auger, 2014). However, Gray (2013) found that average climate variables were a better predictor of large-scale outbreak duration and severity than forest composition. Detailed data on natural enemies and forest composition could improve predictions.

\section{Conclusions}

Dramatic spruce budworm outbreaks have inspired the development of many models, including statistical and simulation models. Here we reinterpreted outbreaks as a metapopulation process so that forest defoliation data could be employed as an indicator of low and high insect densities. It is beneficial to estimate parameters on the same scale at which outbreaks are observed, because emergent outbreak characteristics may not be predicted from local mechanisms (Fleming et al., 2002). Simple metapopulation models are as useful as complex local population models, especially when the ecological mechanisms are hard to parameterize or simply unknown (Harrison 
451

452

et al., 2011). Simple models are also more applicable because of their ease of use and generality, and can easily be extended to other species. The relative importance of defoliation spread is one of the main hurdles for understanding outbreaks (Myers and Cory, 2013) and here we present a method to quantify defoliation spread kernels from aerial survey data. The landscape-scale of our processbased metapopulation model makes it relevant for the development of large-scale forestry and budworm population management practices. Pest management considers pests as immobile, possibly preventing effective management (Tscharntke et al., 2007). Because metapopulation models can be analogous to epidemiological models (Grenfell and Harwood, 1997), widely tested epidemiological techniques to minimize transmission can be considered. Our metapopulation model could be used to generate management strategies that minimize defoliation spread and thus outbreaks, such as spatially-explicit harvesting or spraying programmes. Moreover, to our knowledge for the first time in nature, results suggest for that spatiotemporal autocorrelation in weather values may generate insect outbreaks.

\section{Acknowledgements}

Thanks to the organizers and participants of the IUFRO workshop 'Climate-Induced Range-Shifts in Boreal Forest Pests,' and the organizers of the CJFR special issue. Funding was provided by the Forest Complexity Modelling programme, Natural Sciences and Engineering Research Council of Canada - Collaborative Research and Training Experience and Discovery programmes, Canada Research Chairs, Université du Quebec à Montréal and a Doctoral Research Fellowship from the Fonds de recherche du Quebec - Nature et technologies. Many thanks to Emily Tissier, Alyssa Butler, Mathieu Bouchard and two anonymous reviewers for comments on the manuscript. We also thank Kevin McCann, Daniel Kneeshaw and Patrick James who reviewed this manuscript. 
473

474

475

476

477

478

479

480

481

482

483

484

485

486

\section{References}

Aukema, B.H., Carroll, A.L., Zheng, Y., Zhu, J., Raffa, K.F., Dan Moore, R., Stahl, K., and Taylor, S.W. 2008. Movement of outbreak populations of mountain pine beetle: Influences of spatiotemporal patterns and climate. Ecography 31: 348-358. doi:10.1111/j.0906-7590.2007.05453.x.

Bestelmeyer, B.T., Herrick, J.E., Brown, J.R., Trujillo, D.A., and Havstad, K.M. 2004. Land management in the American southwest: a state-and-transition approach to ecosystem complexity. Environ. Manage. 34: 38-51. doi:10.1007/s00267-004-0047-4.

Bouchard, M. and Auger, I. 2014. Influence of environmental factors and spatio-temporal covariates during the initial development of a spruce budworm outbreak. Landsc. Ecol. 29(1): 111-126. doi: $10.1007 /$ s10980-013-9966-x.

Boyd, I.L., Freer-Smith, P.H., Gilligan, C.A., and Godfray, H.C.J. 2013. The consequence of tree pests and diseases for ecosystem services. Science 342(2013): 1235773. doi:10.1126/science.1235773.

Buckland, S.T. and Elston, D.A. 1993. Empirical Models for the Spatial Distribution of Wildlife. J. Appl. Ecol. 30(3): 478. doi:10.2307/2404188.

Burns, R.M. and Honkala, B.H. 1990. Silvics of North America: 1. Conifers; 2. Hardwoods. vol.2. Agriculture Handbook 654. U.S. Department of Agriculture, Forest Service, Washington, DC.

Candau, J.N. and Fleming, R.A. 2005. Landscape-scale spatial distribution of spruce budworm defoliation in relation to bioclimatic conditions. Can. J. For. Res. 35: 2218-2232. doi:10.1139/X05-078.

Cappuccino, N., Lavertu, D., Bergeron, Y., and Régnière, J. 1998. Spruce budworm impact, abundance and parasitism rate in a patchy landscape. Oecologia 114(2): 236-242. doi:10.1007/ s004420050441. 
494

495

Cooke, B.J., Nealis, V.G., and Régnière, J. 2007. Insect defoliators as periodic disturbances in northern forest ecosystems. In Plant Disturb. Ecol. Process Response, edited by E. Johnson and K. Miyanishi, Elsevier, Burlington, Massachusetts, USA., pp. 487-525.

Cressie, N. and Wikle, C. 2011. Statistics for Spatio-Temporal Data. Wiley.

Cuddington, K.M., Fortin, M.j., Gerber, L.R., Hastings, A., Liebhold, A.M., O'Connor, M., and Ray, C. 2013. Process-based models are required to manage ecological systems in a changing world. Ecosphere 4(2): 1-12. doi:10.1890/ES12-00178.1.

Durrett, R. and Levin, S.A. 1994. The importance of being discrete (and spatial). Theor. Popul. Biol. 46(3): 363-394. doi:10.1006/tpbi.1994.1032.

Fleming, R.A. 2000. Climate change and insect disturbance regimes in Canada's boreal forests. World Resour. Rev. 12(3): 521-555.

Fleming, R.A., Barclay, H.J., and Candau, J.N. 2002. Scaling-up an autoregressive time-series model (of spruce budworm population dynamics) changes its qualitative behavior. Ecol. Modell. 149(12): 127-142. doi:10.1016/S0304-3800(01)00519-1.

Gilbert, B. and O'Connor, M.I. 2013. Climate change and species interactions: Beyond local communities. Ann. N. Y. Acad. Sci. 1297: 98-111. doi:10.1111/nyas.12149.

Gonzalez, A. and Holt, R.D. 2002. The inflationary effects of environmental fluctuations in sourcesink systems. Proc. Natl. Acad. Sci. 99(23): 14872-14877.

Gray, D.R. 2013. The influence of forest composition and climate on outbreak characteristics of the spruce budworm in eastern Canada. Can. J. For. Res. 1195: 1181-1195.

Greenbank, D. 1956. The role of climate and dispersal in the inititiation of outbreaks of the spruce budworm in New Brunswick. 1. The role of climate. Can. J. Zool. 34: 453-476. https://mc06.manuscriptcentral.com/cjfr-pubs 
Greenbank, D. 1980. Spruce budworm (Lepidoptera: Tortricidae) moth flight and dispersal: new understanding from canopy observations, radar, and aircraft. Mem. Entomol. Soc. Canada 112: 1-49. doi:10.4039/entm112110fv.

Grenfell, B.T. and Harwood, J. 1997. (Meta)population dynamics of infectious diseases. Trends Ecol. \& Evol. 12: 395-399.

Griffith, D.A. and Peres-Neto, P.R. 2006. Spatial modeling in Ecology: the flexibility of the eigenfunction spatial analyses. Ecology 87(10): 2603-2613. doi:10.1890/0012-9658(2006)87[2603:SMIETF]2. $0 . \mathrm{CO} ; 2$.

Hanski, I. 1998. Metapopulation Dynamics. Nature 396(6706): 41-49. doi:10.1038/23876.

Harrison, P.J., Hanski, I., and Ovaskainen, O. 2011. Bayesian state-space modeling of metapopulation dynamics in the Glanville fritillary butterfly. Ecol. Monogr. 81(4): 581-598. doi:10.1890/11-0192.1.

James, P.M.A., Cooke, B.J., Brunet, B.M.T., Lumley, L.M., and Felix, A. 2015. Life-stage differences in spatial genetic structure in an irruptive forest insect : implications for dispersal and spatial synchrony. Mol. Ecol. 24: 296-309. doi:10.1111/mec.13025.

Kärvemo, S., Johansson, V., Schroeder, M., and Ranius, T. 2016. Local colonization-extinction dynamics of a tree-killing bark beetle during a large-scale outbreak. Ecosphere 7(3): 1-14. doi: 10.1002/ecs2.1257.

Keeling, M.J. and Rohani, P. 2008. Modeling infectious diseases in humans and animals. Princeton University Press, Princeton, NJ.

Keeling, M.J., Woolhouse, M.E.J., May, R.M., Davies, G., and Grenfell, B.T. 2003. Modelling vaccination strategies against foot-and-mouth disease. Nature 421(6919): 136-42. doi:10.1038/ nature01343. 
Kendall, B.E., Briggs, C.J., Murdoch, W.W., Turchin, P., Ellner, S.P., McCauley, E., Nisbet, R.M., and Wood, S.N. 1999. Why do populations cycle? A synthesis of statistical and mechanistic modeling approaches. Ecology 80: 1789-1805. doi:10.1890/0012-9658(1999)080[1789:WDPCAS]2.0.CO;2.

Kermack, W.O. and McKendrick, A.G. 1927. A Contribution to the Mathematical Theory of Epidemics. Proc. R. Soc. A Math. Phys. Eng. Sci. 115(772): 700-721. doi:10.1098/rspa.1927.0118.

Kissling, W.D. and Carl, G. 2008. Spatial autocorrelation and the selection of simultaneous autoregressive models. Glob. Ecol. Biogeogr. 17: 59-71. doi:10.1111/j.1466-8238.2007.00334.x.

Koenig, W.D. 2002. Global patterns of environmental synchrony and the Moran effect. Ecography 25(3): 283-288.

Levins, R. 1969. Some Demographic and Genetic Consequences of Environmental Heterogeneity for Biological Control. Bull. Ecol. Soc. Am. 15(3): 237-240.

Liebhold, A.M., Koenig, W.D., and Bjørnstad, O.N. 2004. Spatial Synchrony in Population Dynamics. Annu. Rev. Ecol. Evol. Syst. 35(1): 467-490. doi:10.1146/annurev.ecolsys.34.011802.132516.

Massie, T.M., Weithoff, G., Kuckländer, N., Gaedke, U., and Blasius, B. 2015. Enhanced Moran effect by spatial variation in environmental autocorrelation. Nat. Commun. 6: 5993 . doi: 10.1038/ncomms6993.

McKenney, D.W., Hutchinson, M.F., Papadopol, P., Lawrence, K., Pedlar, J., Campbell, K., Milewska, E., Hopkinson, R.F., Price, D.T., and Owen, T. 2011. Customized Spatial Climate Models for North America. Publicly available at gmaps.nrcan.gc.ca/cl_p/climatepoints.php. Bull. Am. Meteorol. Soc. 92(12): 1611-1622. doi:10.1175/2011BAMS3132.1.

MFFP 2014. Ministère des Forêts de la Faune et des Parcs Direction de la protection des forêts. Service 
de la gestion des Ravageurs. Donnees sur les perturbations naturelles: Tordeuse des bourgeons de l'épinette. Données ouvertes .

Moilanen, A. 1999. Patch occupancy models of metapopulation dynamics: efficient parameter estimation using implicit statistical inference. Ecology 80(3): 1031-1043.

Moilanen, A. 2004. SPOMSIM: Software for stochastic patch occupancy models of metapopulation dynamics. Ecol. Modell. 179: 533-550. doi:10.1016/j.ecolmodel.2004.04.019.

Moran, P.A.P. 1953. The statistical analysis of the Canadian lynx cycle. I. Structure and prediction. Aust. J. Zool. 1(2): 291-298.

Morin, H., Jardon, Y., and Gagnon, R. 2007. Relationship Between Spruce Budworm Outbreaks and Forest Dynamics in Eastern North America. In Plant Disturb. Ecol. Process Response, edited by E. Johnson and K. Miyanishi, Elsevier, New York, pp. 555-577.

Myers, J.H. and Cory, J.S. 2013. Population Cycles in Forest Lepidoptera Revisited. Annu. Rev. Ecol. Evol. Syst. 44(1): 565-592. doi:10.1146/annurev-ecolsys-110512-135858.

Peltonen, M., Liebhold, A.M., Bjørnstad, O.N., and Williams, D.W. 2002. Spatial synchrony in forest insect outbreaks: roles of regional stochasticity and dispersal. Ecology 83: 3120-3129. doi:10. 1890/0012-9658(2002)083[3120:SSIFIO]2.0.CO;2.

Peters, D.P.C., Herrick, J.E., Urban, D.L., Gardner, R.H., and Breshears, D.D. 2004. Strategies for ecological extrapolation. Oikos 106(3): 627-636. doi:10.1111/j.0030-1299.2004.12869.x.

Preisler, H.K., Hicke, J.A., Ager, A.A., and Hayes, J.L. 2012. Climate and weather influences on spatial temporal patterns of mountain pine beetle populations in Washington and Oregon. Ecology 93(11): 2421-2434. doi:10.1890/11-1412.1. 
Pureswaran, D.S., Johns, R., Heard, S.B., and Quiring, D. 2016. Paradigms in Eastern Spruce Budworm (Lepidoptera: Tortricidae) Population Ecology: A Century of Debate. Environ. Entomol. p. nvw103. doi:10.1093/ee/nvw103.

Régnière, J., Delisle, J., Pureswaran, D.S., and Trudel, R. 2013. Mate-finding Allee effect in spruce budworm population dynamics. Entomol. Exp. Appl. 146: 112-122. doi:10.1111/eea.12019.

Régnière, J. and Nealis, V.G. 2007. Ecological mechanisms of population change during outbreaks of the spruce budworm. Ecol. Entomol. 32(5): 461-477. doi:10.1111/j.1365-2311.2007.00888.x.

Régnière, J., Powell, J., Bentz, B., and Nealis, V.G. 2012. Effects of temperature on development, survival and reproduction of insects: experimental design, data analysis and modeling. J. Insect Physiol. 58(5): 634-47. doi:10.1016/j.jinsphys.2012.01.010.

Robert, L.e., Kneeshaw, D., and Sturtevant, B.R. 2012. Effects of forest management legacies on spruce budworm ( Choristoneura fumiferana ) outbreaks. Can. J. For. Res. 475: 463-475. doi: $10.1139 /$ X2012-005.

Roland, J. and Taylor, P. 1997. Insect parasitoid species respond to forest structure at different spatial scales. Nature 386(6626): 710-713. doi:10.1038/386710a0.

Royama, T. 1984. Population Dynamics of the Spruce Budworm Choristoneura fumiferana. Ecol. Monogr. 54: 429-462.

Ruokolainen, L., Lindén, A., Kaitala, V., and Fowler, M.S. 2009. Ecological and evolutionary dynamics under coloured environmental variation. Trends Ecol. \& Evol. 24(10): 555-63. doi: 10.1016/j.tree.2009.04.009.

Sturtevant, B.R., Achtemeier, G.L., Charney, J.J., Anderson, D.P., Cooke, B.J., and Townsend, P.A. 2013. Long-distance dispersal of spruce budworm ( Choristoneura fumiferana Clemens ) in Minhttps://mc06.manuscriptcentral.com/cjfr-pubs 
nesota ( USA ) and Ontario ( Canada ) via the atmospheric pathway. Agric. For. Meteorol. 168: $186-200$.

Sturtevant, B.R., Cooke, B.J., Kneeshaw, D., and Maclean, J.E. 2015. Modeling Insect Disturbance Across Forested Landscapes: Insights from the Spruce Budworm. In Simul. Model. For. Landsc. Disturbances, edited by A.H. Perera, B.R. Sturtevant, and L.J. Buse, Springer, pp. 1-321. doi:10. 1007/978-3-319-19809-5.

Tian, H., Stige, L.C., Cazelles, B., Kausrud, K.L., Svarverud, R., Stenseth, N.C., and Zhang, Z. 2011. Reconstruction of a 1,910-y-long locust series reveals consistent associations with climate fluctuations in China. Proc. Natl. Acad. Sci. 108(6): 14521-14526. doi:10.1073/pnas.1100189108.

Tscharntke, T., Bommarco, R., Clough, Y., Crist, T.O., Kleijn, D., Rand, T.A., Tylianakis, J.M., Van Nouhuys, S., and Vidal, S. 2007. Conservation biological control and enemy diversity on a landscape scale. Biol. Control 43: 294-309. doi:10.1016/j.biocontrol.2007.08.006.

Vasseur, D.A. 2007. Environmental colour intensifies the Moran effect when population dynamics are spatially heterogeneous. Oikos 116(10): 1726-1736. doi:10.1111/j.2007.0030-1299.16101.x.

Veran, S., Simpson, S.J., Sword, G.A., Deveson, E., Piry, S., Hines, J.E., and Berthier, K. 2014. Modeling spatiotemporal dynamics of outbreaking species: influence of environment and migration in a locust. Ecology 96(3): 737-748. doi:10.1890/14-0183.1.

Williams, D.W. and Liebhold, A.M. 2000. Spatial synchrony of spruce budworm outbreaks in eastern North America. Ecology 81(10): 2753-2766.

Wilmers, C.C., Post, E., and Hastings, A. 2007. A perfect storm: the combined effects on population fluctuations of autocorrelated environmental noise, age structure, and density dependence. Am. Nat. 169(5): 673-83. doi:10.1086/513484. 
${ }_{624}$ Yackulic, C.B., Nichols, J.D., Reid, J., and Der, R. 2015. To predict the niche, model colonization and ${ }_{625}$ extinction. Ecology 96(1): 16-23. doi:10.1890/14-1361.1.

${ }_{626}$ Zhou, G. and Liebhold, A.M. 1995. Forecasting the spatial dynamics of gypsy moth outbreaks using ${ }_{627}$ cellular transition models. Landsc. Ecol. 10(3): 177-189. doi:10.1007/BF00133030. 


\section{Tables}

Table 1: The potential weather variables used in the analysis and their time lags tested. The interpolated values covered each cell in the study area (Fig. 1 b) from 1965- 2013. From McKenney et al., 2011. Bottom five lines, the types of weather randomizations.

weather Variable

Time lag

Max/min temperature of warmest/coldest period

$\mathrm{Max} / \mathrm{min}$ temperature of coldest month

1 year before

Max/min temperature of spring (April-May)

2 years before

Max/min temperature of summer (June-July)

3 years before

Length of growing season (Growing Degree Days)

Total Precipitation

Elevation (constant in time)

Original time, original space

Random time, original space

Original time, random space

Random time, random space

Average time and space (constant) 
Table 2: Model selection results on defoliation FI transitions. The three best climate variables were summer maximum temperature $t-3$, summer minimum temperature $t-3$ and number of growing degree days $t-2$. The variables had third-degree response terms. $\mathrm{K}=$ the number of variables.

\begin{tabular}{l|rrrrrr}
\hline & K & AIC & deltaAIC & pseudo-r^2 & random residuals & temporal residuals \\
\hline all climate + dispersal & 79 & 19475 & 0 & 0.425 & 2439 & 1375 \\
all climate & 76 & 24374 & 4899 & 0.300 & 2842 & 1582 \\
3 climate + dispersal & 13 & 27599 & 8124 & 0.214 & 3390 & 1387 \\
1 climate x dispersal & 16 & 28084 & 8609 & 0.186 & 3610 & 1395 \\
1 climate + dispersal & 7 & 28860 & 9386 & 0.175 & 3558 & 1384 \\
dispersal & 4 & 28889 & 9414 & 0.168 & 3610 & 1378 \\
3 climate (with interactions) & 64 & 29708 & 10234 & 0.156 & 3329 & 1575 \\
global dispersal & 4 & 30548 & 11073 & 0.120 & 3688 & 1490 \\
3 climate & 10 & 32076 & 12602 & 0.066 & 3721 & 1581 \\
1 climate & 4 & 33678 & 14203 & 0.015 & 3868 & 1572 \\
latitude x longitude & 16 & 34596 & 15121 & 0.013 & 3751 & 1562 \\
\hline
\end{tabular}




\section{Figure Captions}

Fig. 1. Left, the proportion of outbreaks over time, expressed as the proportion of cells in the defoliated state. Right, the geographic extent of the study area in Quebec province, Eastern Canada. Each square is a 'cell' (6 446 in total) and the colour indicates its state: Dark grey (red online) is defoliated/defoliated, grey (green online) is forest non-defoliated cell in 2015. Grey areas were never defoliated and excluded from the analysis.

Fig. 2. Schematic representation of a metapopulation approach to forest insect outbreaks, indicating the forest $F$ and defoliated $I$ states. The arrows indicate the parameters that set the state transitions defoliation $F \rightarrow I$ and mortality $I \rightarrow F$.

Fig. 3. Map of $\alpha K$ calculated from the cells defoliated in 2013 (in dark grey, red online). The defoliation spread kernel $K$ was calculated using the negative exponential kernel with $\delta=10$.

Fig. 4. The simulated proportion of defoliated cells with different types of spatiotemporally autocorrelated weather. Dotted lines indicates the observed outbreaks, and each thin line indicates the proportion of defoliated landscape during one simulated outbreak.

Fig. 5. Summary results from simulation model. Horizontal dotted lines indicate the values in the observed outbreaks. Each plot shows the values from 100 simulations. Boxes encompass the $25 \%$ - 75\% quartiles of the data, with the median indicated by the thick line through the centre of each box. Whiskers extending from the box encompass the $95 \%$ quartiles, and extreme observations are shown as circles. a, Maximum proportion of defoliated area. $b$, Standard deviation in the simulated proportion of defoliated area I. c, Temporal autocorrelation with a 1 year time lag. d, Range of positive temporal autocorrelation (in years). e, Maximum number of contiguous outbreak clusters per simulation. f, Largest contiguous outbreak cluster size per simulation (number of cells). 


\section{Figures}
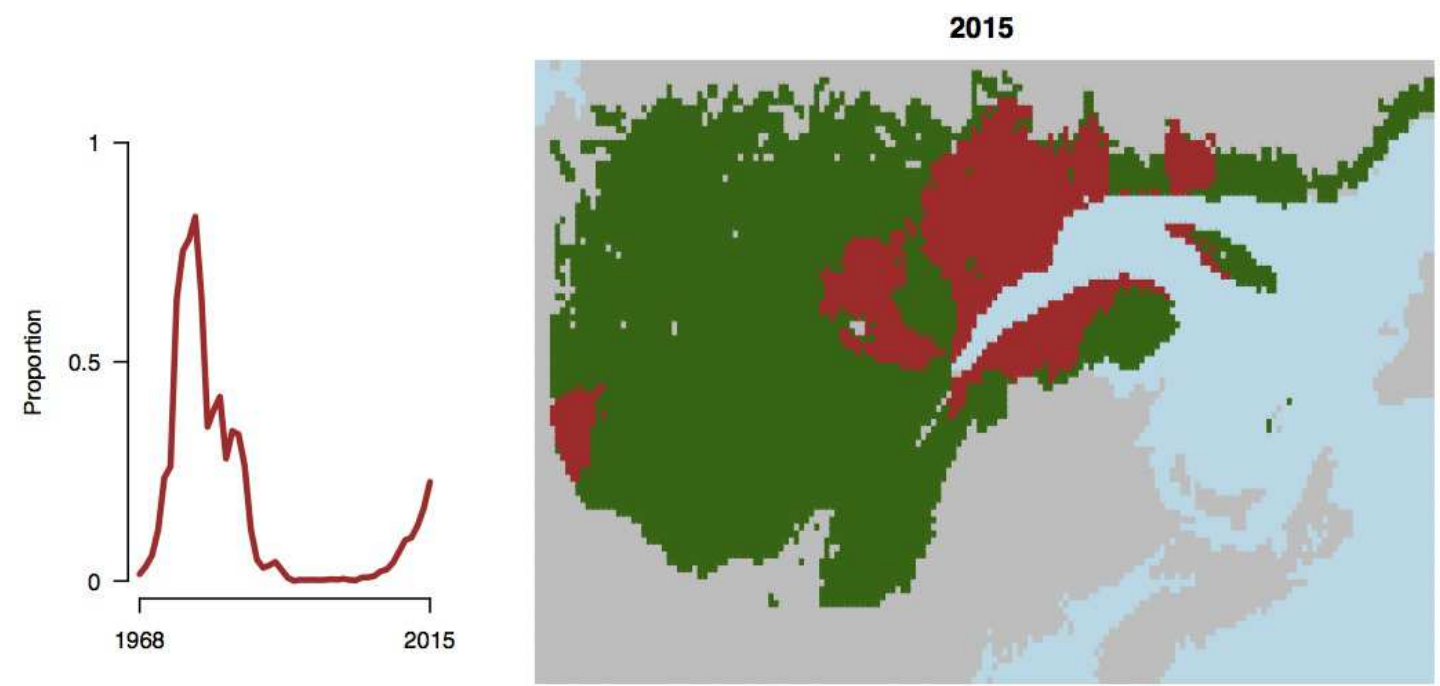

Fig. 1: Left, the proportion of outbreaks over time, expressed as the proportion of cells in the defoliated state. Right, the geographic extent of the study area in Quebec province, Eastern Canada. Each square is a 'cell' (6 446 in total) and the colour indicates its state: Dark grey (red online) is defoliated/defoliated, grey (green online) is forest non-defoliated cell in 2015. Grey areas were never defoliated and excluded from the analysis. 


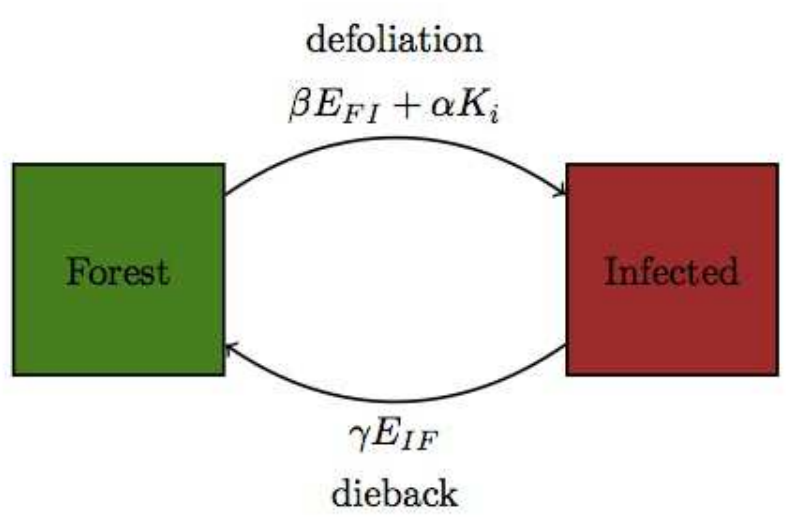

$$
\begin{aligned}
\beta & =\text { probability of spontaneous infection } \\
\alpha & =\text { probability of infection spread } \\
\gamma & =\text { probability of a dieback to forest } \\
E_{F I} & =\text { predicted climate suitability of defoliation } \\
E_{I F} & =\text { predicted climate suitability of dieback } \\
K_{i} & =\text { dispersal kernel }
\end{aligned}
$$

Fig. 2: Schematic representation of a metapopulation approach to forest insect outbreaks, indicating the forest $F$ and defoliated $I$ states. The arrows indicate the parameters that set the state transitions defoliation $F \rightarrow I$ and mortality $I \rightarrow F$. 


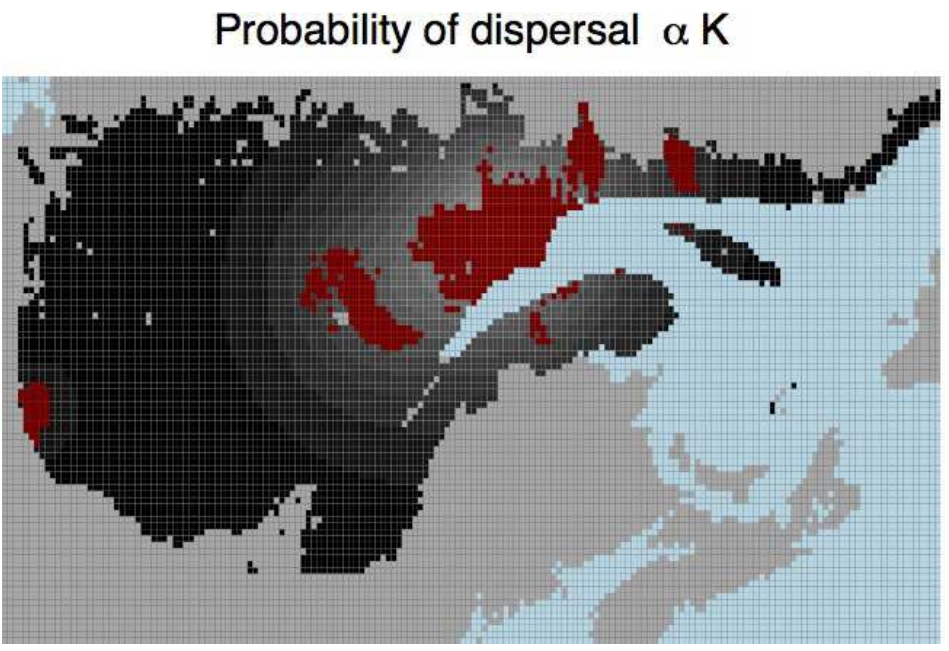

Predicted probability of dispersal

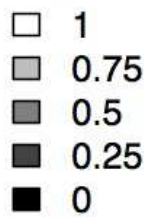

Fig. 3: Map of $\alpha K$ calculated from the cells defoliated in 2013 (in dark grey, red online). The defoliation spread kernel $K$ was calculated using the negative exponential kernel with $\delta=10$. 
a) Spatio-temporal autocorrelation (original climate)

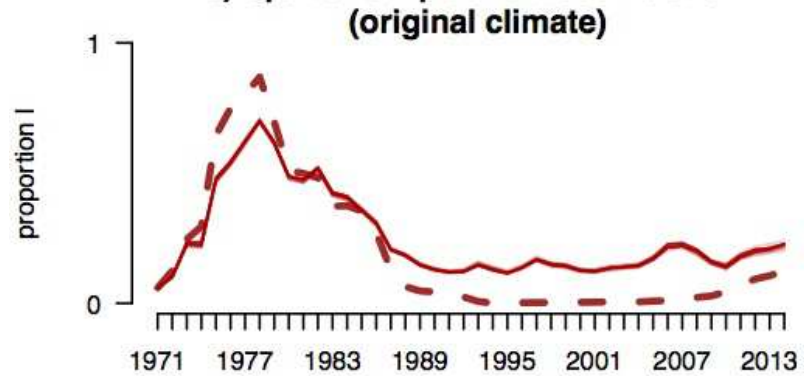

c) Only spatial autocorrelation

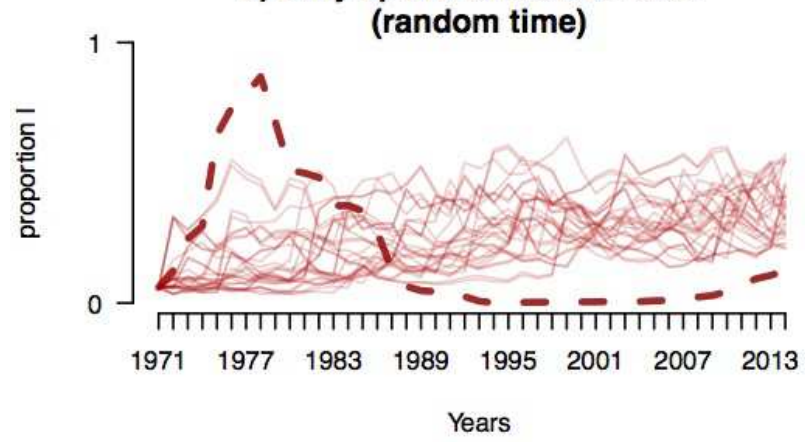

e) Constant climate

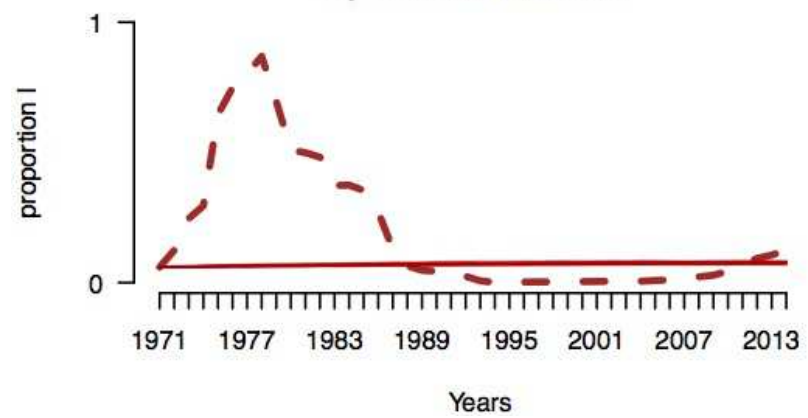

b) Only temporal autocorrelation

(random space)

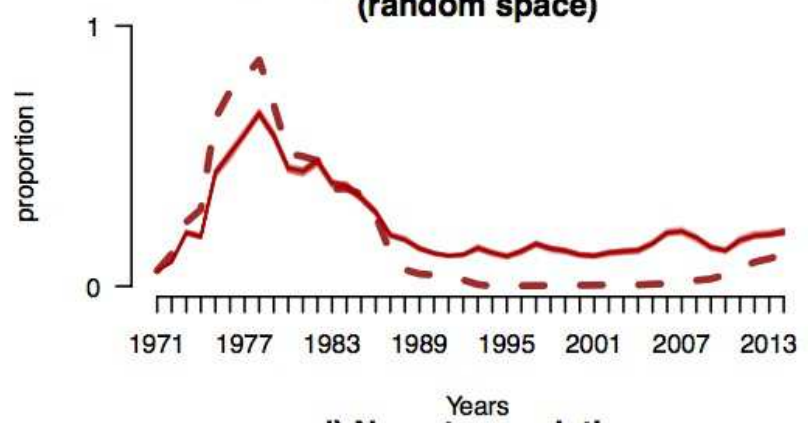

d) No autocorrelation (random climate)

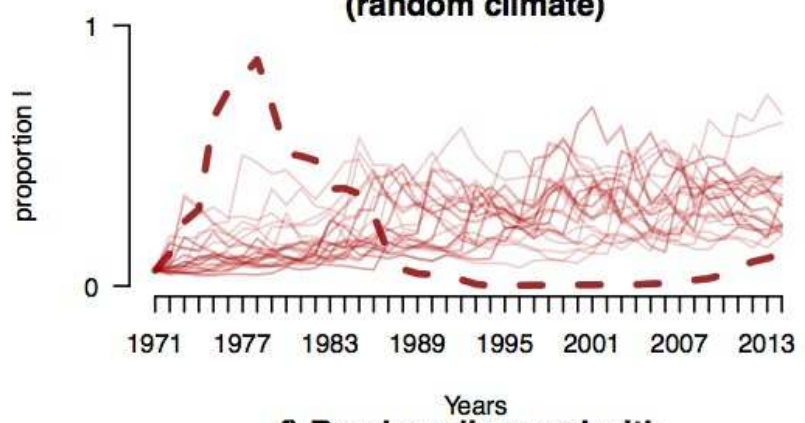

f) Random dispersal with Spatio-temporal autocorrelation

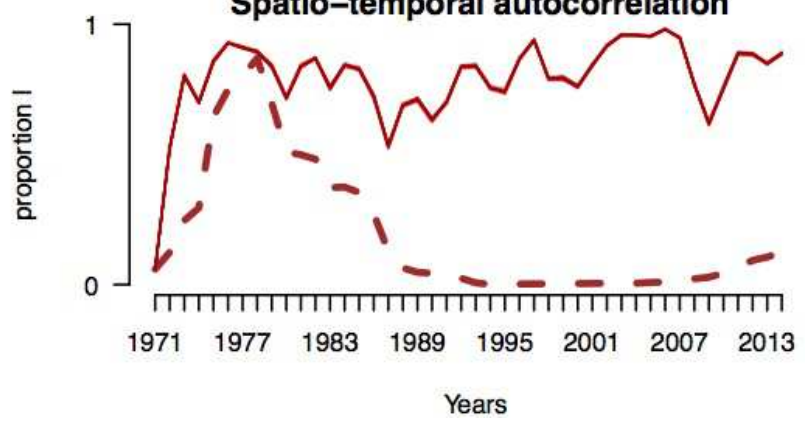

Fig. 4: The simulated proportion of defoliated cells with different types of spatiotemporally autocorrelated weather. Dotted lines indicates the observed outbreaks, and each thin line indicates the proportion of defoliated landscape during one simulated outbreak. 


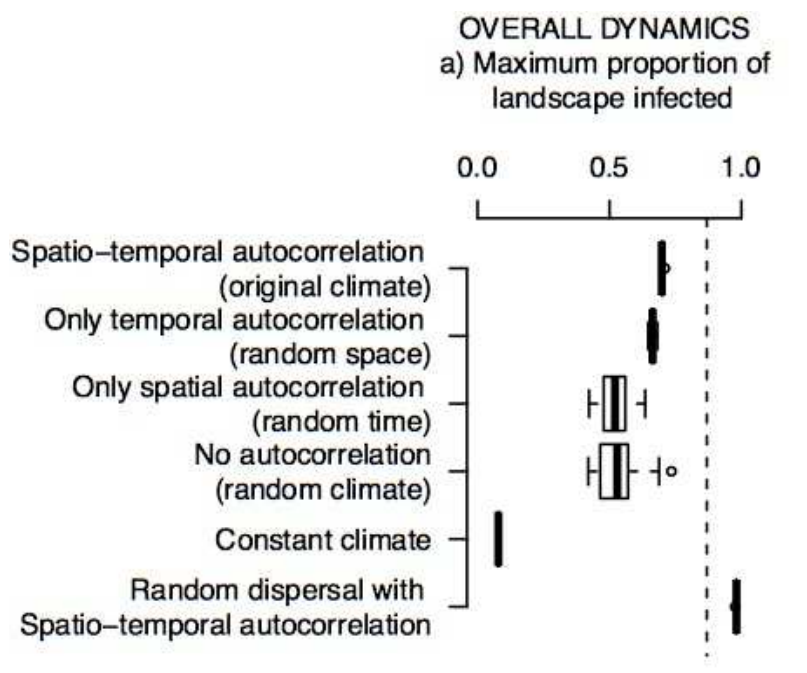

b) S.d in proportion of landscape infected

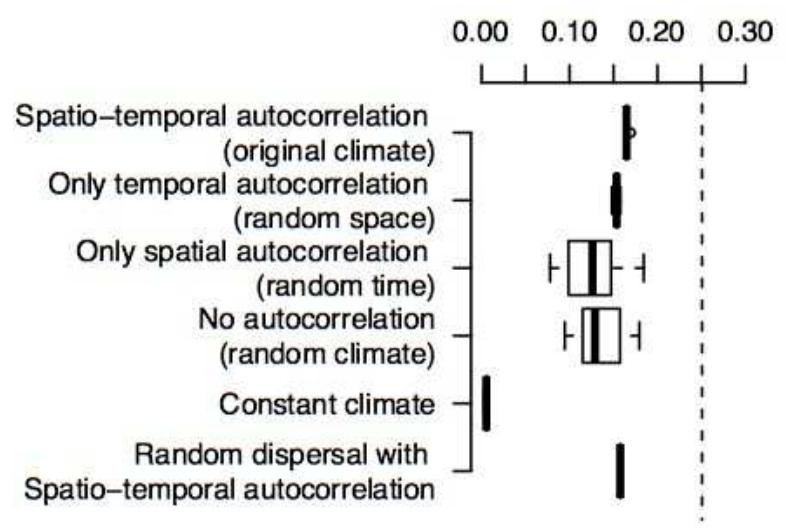

TEMPORAL DYNAMICS

c) Temporal autocorrelation with timelag 1 year

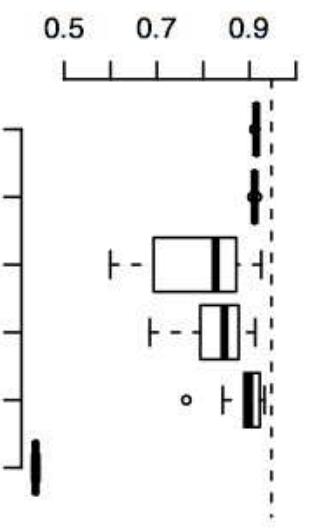

d) Range of positive temporal autocorrelation (years)

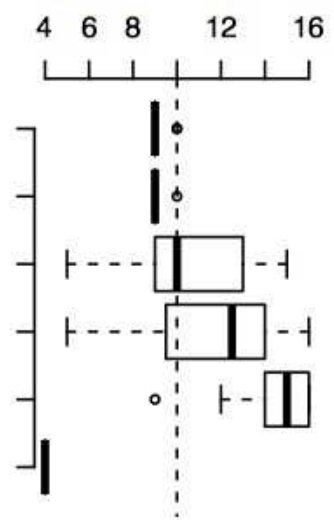

SPATIAL DYNAMICS

e) Maximum number of clusters (contigous infected patches)

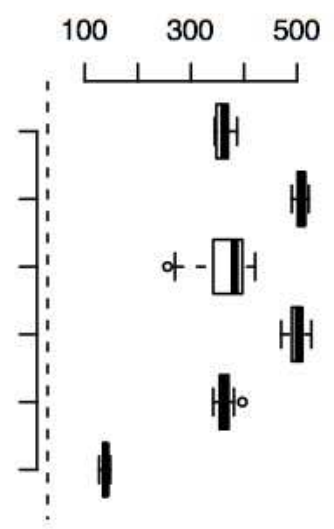

f) Maximum cluster size (number of patches)

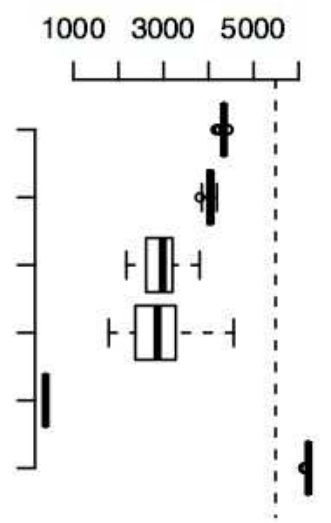

Fig. 5: Summary results from simulation model. Horizontal dotted lines indicate the values in the observed outbreaks. Each plot shows the values from 100 simulations. Boxes encompass the $25 \%$ - 75\% quartiles of the data, with the median indicated by the thick line through the centre of each box. Whiskers extending from the box encompass the $95 \%$ quartiles, and extreme observations are shown as circles. a, Maximum proportion of defoliated area. $b$, Standard deviation in the simulated proportion of defoliated area I. c, Temporal autocorrelation with a 1 year time lag. d, Range of positive temporal autocorrelation (in years). e, Maximum number of contiguous outbreak clusters per simulation. f, Largest contiguous outbreak cluster size per simulation (number of cells). 


\title{
More than Moran: Coupling statistical and simulation models to understand how defoliation spread and weather variation drive outbreak dynamics
}

\author{
March 7, 2017
}

\section{Parameter results}

The weather prediction $E_{F I}$ was low even in the most suitable weather ( $\beta=0.008$, Figure S7).

The mean weather prediction of infections $E_{F I}$ was higher along the coast and in the south. In models with only the best weather variable, the probability of spontaneous outbreaks was highest at moderate maximum summer temperatures, two years previously (Figure S7c).

The mean defoilation mortality to forest $E_{I F}$ was more probable on the geographic range edges, meaning that the outbreaks die back faster in unsuitable weather closer to the range edges ( $\gamma$, Figure S7b). In unsuitable weather, (i.e. in extreme spring maximum temperatures) the probability of an defoliated forest becoming forest in the next year was close to one (Figure S7 d).

Dispersal probabilities were highest at around $K_{i}=0.5$ (Figure S7 e). Dispersal probabilities were also affected by weather, so in suitable weather the probability of defoliation spread can reach 1 (thin lines in Figure S7 e).

Multiple weather variables influence the transition of outbreaks. Summer and spring temperatures generally had a large effect on infection probabilities. Temperature during the growth period determines the insect-temperature response curves in the laboratory (Régnière 1987). In contrast to a similar smaller-scale study with linear weather relationships, precipitation and elevation variables were not selected (Bouchard and Auger 2014). Temperature variables were selected here because our study spanned a wide environmental gradient, to which nonlinear temperature responses fit better. Non-parametric models have suggested that temperature determines cumulative budworm damage (Candau and Fleming 2011; Gray 2013), and here we show that temperature also affects infection transitions.

\section{Analysis of spatial autocorrelation in the statistical model}

It was necessary to separate the relative contribution of defoliation spread and (multiple) weather variables to outbreaks, so predictions were divided up to defoliation spread- and weather-dependent partial predictions. Weather predictions $E_{F I}$ represented the contribution of multiple weather variables to the probability of transition. The total probability of transition comes from adding the weather predictions $E_{F I}$ and neighbourhood infection $K$ :

$$
\text { Probability }\{F \rightarrow I\}_{i}=E_{F I}+\alpha_{1} K+\alpha_{2} K^{2}+\alpha_{3} K^{3}
$$

We analyzed if a higher autocorrelation in $E_{F I}$ was associated with more successful infection transitions. We examined the effect of autocorrelation in $E_{F I}$ in time. We assessed if a series of years with suitable weather for outbreaks occurred during large-scale outbreaks, i.e. in years with temporally autocorrelated predictions. To calculate the temporal autocorrelation of predictions, we first calculated the annual mean of infection $E_{F I}$ and mortality $E_{I F}$ predictions. We then estimated during which years the predictions were asynchronous (phase coherence in wavelet cross-correlation, R-package biwavelet, Gouhier and Grinsted 2012).

The effect of temporal autocorrelation in weather predictions $E$ on infections shows that the outbreak in 1971-1980 occurred at the same time as a high weather predictions $E_{F I}$, and low average weather predictions $E_{F I}$. This combination led to many new infection transitions that stayed defoliated longer (Figure S9 a). Infection and mortality transition probabilities were asynchronous for several years at the beginning of the outbreak (not significant, Figure S9 b). However, the probabilities were also asynchronous between outbreaks. 


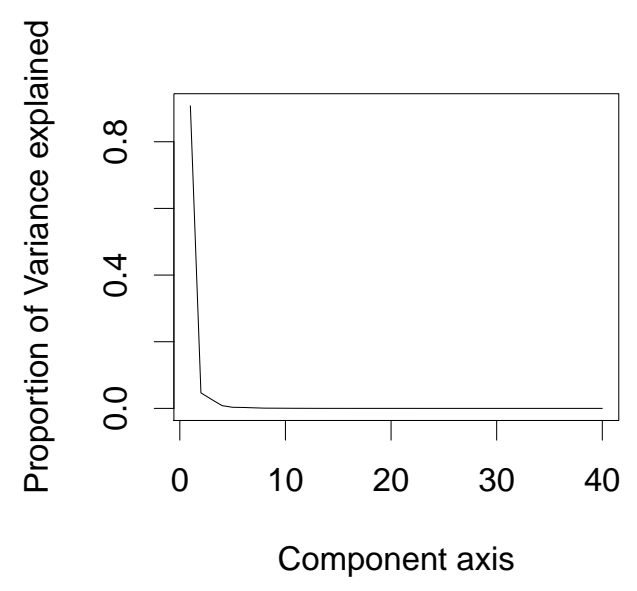

Figure S1: Proportion of variance explained by the first 25 axes of a PCA on the defoliation spread variables K.

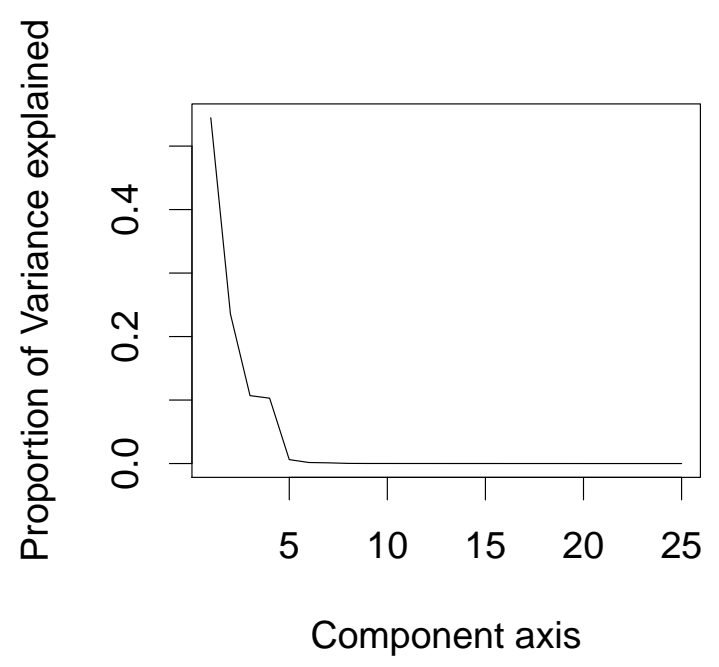

Figure S2: Proportion of variance explained by the first 25 axes of a PCA on the weather variables. 

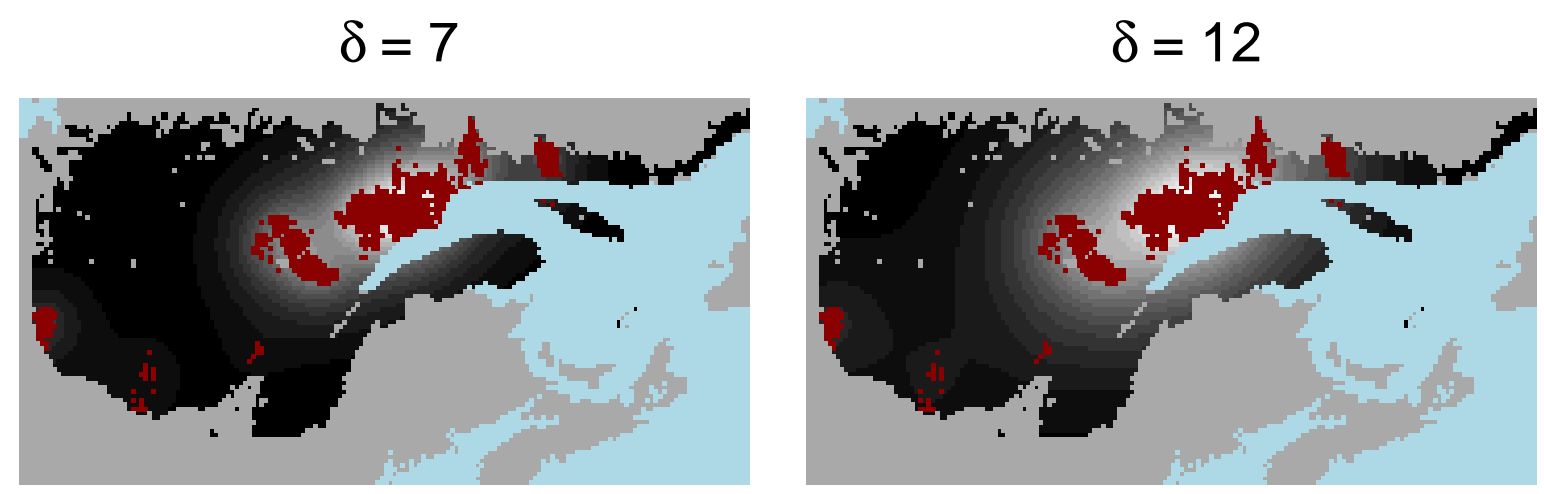

$$
\delta=17
$$
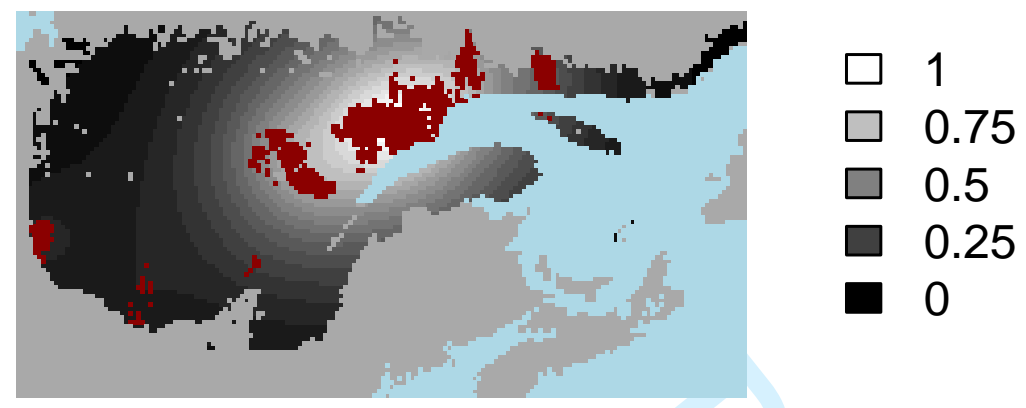

Figure S3: Examples of K with different values for $\delta$, the effect of distance to defoliated cells.

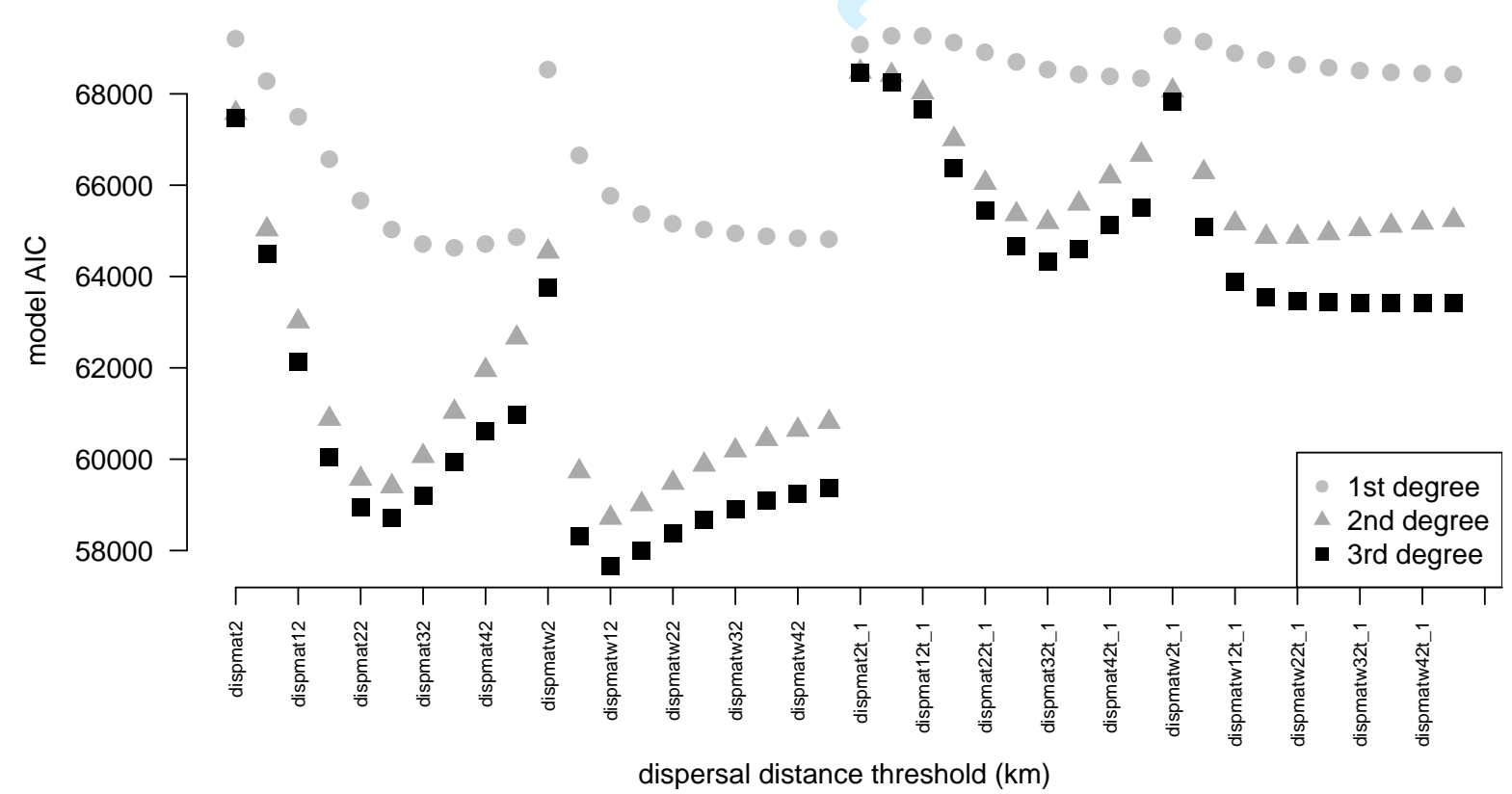

Figure S4: The likelihood profile from selection of the best defoliation spread kernel $K_{i}$ with first, second and third degree response functions. The estimated AIC with defoliation spread kernels calculated with different $\delta$ values, from a forwards stepwise logistic regression. The autologistic and negative exponential kernels are both tested with infection distributions from one and two years previously. 


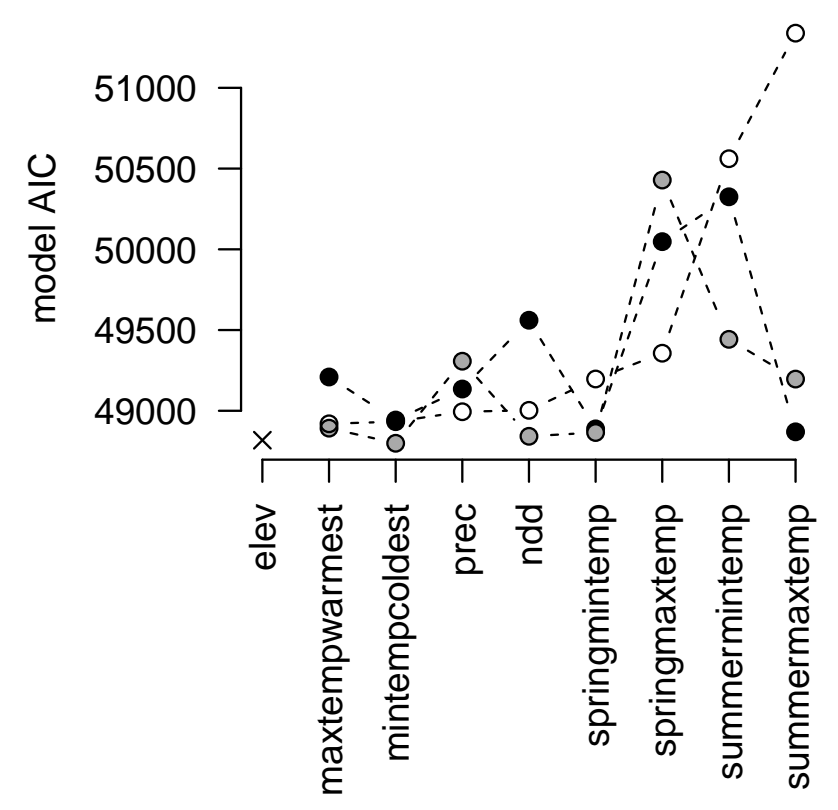

Figure S5: Selection of best weather variable. AIC results of backward model selection results on FI infection transitions, third-degree response terms.

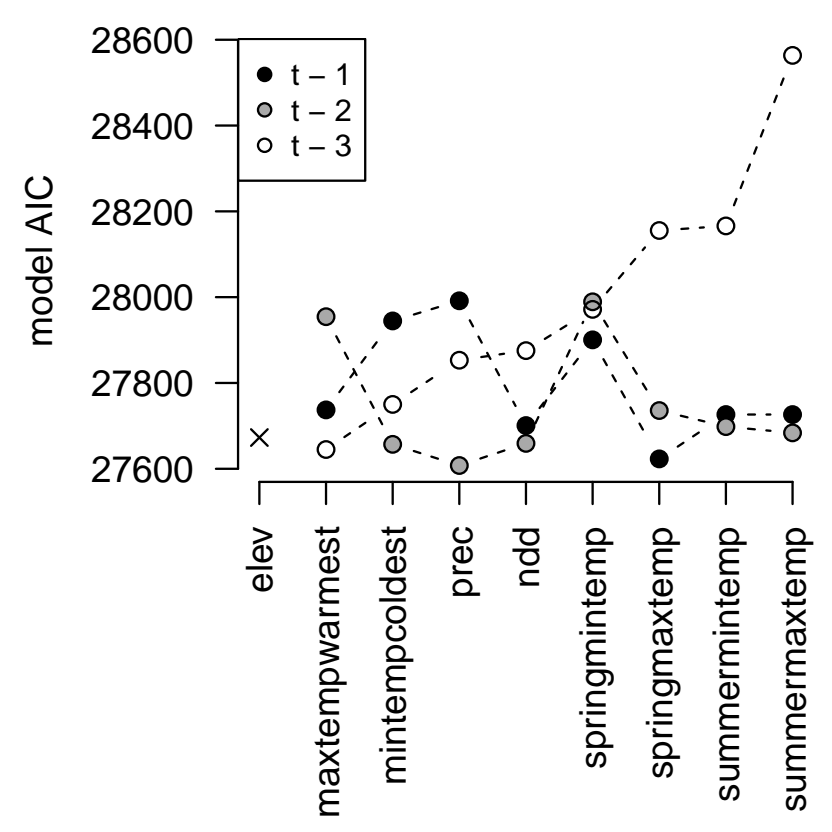

Figure S6: AIC results, backward selection. Model selection results on IM transitions, response terms with 3 degrees and estimated on all data. 
Table S1: AIC results, backward selection. Model selection results on FI transitions, with 1,2 and 3-degree response terms and estimated on all data. Each AIC is obtained when carrying out a glm with all weather variables except that one. The variable when removed results in the highest AIC is the most important variable.

\begin{tabular}{|c|c|c|c|c|c|c|}
\hline & AIC, degree 1 & deltaAIC, degree 1 & AIC, degree 2 & deltaAIC, degree 2 & AIC, degree 3 & deltaAIC, degree 3 \\
\hline summermaxtempt_3 & 61162 & 0 & 54267 & 0 & 51339 & 0 \\
\hline summermintempt_3 & 59273 & 1889 & 53039 & 1228 & 50561 & 778 \\
\hline nddt_2 & 58068 & 3094 & 52490 & 1777 & 50429 & 910 \\
\hline summermintempt_1 & 59091 & 2071 & 53315 & 952 & 50325 & 1014 \\
\hline nddt_1 & 58654 & 2508 & 52693 & 1574 & 50048 & 1291 \\
\hline springmaxtempt_1 & 58012 & 3150 & 51784 & 2483 & 49561 & 1778 \\
\hline summermintempt_2 & 57908 & 3254 & 51704 & 2563 & 49443 & 1896 \\
\hline nddt_3 & 57819 & 3342 & 51791 & 2476 & 49357 & 1982 \\
\hline maxtempwarmestt_2 & 58884 & 2278 & 52058 & 2209 & 49307 & 2032 \\
\hline springmintempt_1 & 57835 & 3327 & 51746 & 2521 & 49209 & 2130 \\
\hline prect_3 & 57774 & 3387 & 51603 & 2664 & 49197 & 2142 \\
\hline summermaxtempt_2 & 58039 & 3122 & 51702 & 2565 & 49196 & 2143 \\
\hline maxtempwarmestt_1 & 58861 & 2301 & 52130 & 2138 & 49135 & 2204 \\
\hline springmaxtempt_3 & 57807 & 3355 & 51391 & 2876 & 49003 & 2336 \\
\hline maxtempwarmestt_3 & 59459 & 1703 & 52166 & 2101 & 48994 & 2345 \\
\hline mintempcoldestt_1 & 57891 & 3271 & 51691 & 2577 & 48943 & 2396 \\
\hline mintempcoldestt_3 & 57793 & 3369 & 51554 & 2713 & 48933 & 2406 \\
\hline springmintempt_3 & 57767 & 3395 & 51815 & 2452 & 48919 & 2419 \\
\hline springmintempt_2 & 57840 & 3321 & 51557 & 2711 & 48892 & 2447 \\
\hline prect_1 & 57775 & 3387 & 51463 & 2804 & 48888 & 2451 \\
\hline summermaxtempt_1 & 57816 & 3345 & 51634 & 2633 & 48870 & 2469 \\
\hline prect_2 & 57872 & 3290 & 51524 & 2743 & 48865 & 2474 \\
\hline springmaxtempt_2 & 57932 & 3230 & 51451 & 2817 & 48842 & 2497 \\
\hline elev & 57768 & 3394 & 51509 & 2758 & 48818 & 2521 \\
\hline mintempcoldestt_2 & 57929 & 3233 & 51536 & 2731 & 48799 & 2540 \\
\hline
\end{tabular}


Table S2: AIC results, backward selection. Model selection results on IF transitions, with 1,2 and 3-degree response terms and estimated on all data. Each AIC is obtained when carrying out a glm with all weather variables except that one. The variable when removed results in the highest AIC is the most important variable.

\begin{tabular}{|c|c|c|c|c|c|c|}
\hline & AIC, degree 1 & deltaAIC, degree 1 & AIC, degree 2 & deltaAIC, degree 2 & AIC, degree 3 & deltaAIC, degree 3 \\
\hline springmintempt_3 & 34641 & 1618 & 29896 & 0 & 28564 & 0 \\
\hline prect_3 & 34744 & 1515 & 28992 & 904 & 28166 & 397 \\
\hline mintempcoldestt_3 & 36259 & 0 & 29393 & 503 & 28155 & 408 \\
\hline summermintempt_1 & 35301 & 958 & 29249 & 647 & 27992 & 572 \\
\hline maxtempwarmestt_2 & 34638 & 1621 & 29200 & 696 & 27989 & 574 \\
\hline maxtempwarmestt_3 & 34804 & 1455 & 29060 & 837 & 27972 & 592 \\
\hline summermaxtempt_2 & 34633 & 1626 & 29009 & 887 & 27955 & 609 \\
\hline nddt_1 & 35141 & 1118 & 29059 & 837 & 27945 & 619 \\
\hline maxtempwarmestt_1 & 34778 & 1481 & 29256 & 641 & 27901 & 663 \\
\hline springmaxtempt_3 & 34659 & 1600 & 29015 & 881 & 27875 & 688 \\
\hline summermintempt_3 & 34649 & 1610 & 28955 & 941 & 27853 & 711 \\
\hline nddt_3 & 34641 & 1618 & 29078 & 818 & 27750 & 814 \\
\hline summermaxtempt_1 & 34718 & 1542 & 28970 & 926 & 27737 & 826 \\
\hline mintempcoldestt_2 & 34813 & 1446 & 28938 & 958 & 27736 & 828 \\
\hline prect_1 & 34679 & 1581 & 28837 & 1059 & 27726 & 837 \\
\hline springmintempt_1 & 34633 & 1627 & 28973 & 923 & 27726 & 837 \\
\hline springmaxtempt_1 & 34635 & 1624 & 28780 & 1116 & 27701 & 863 \\
\hline prect_2 & 34684 & 1575 & 28836 & 1060 & 27698 & 865 \\
\hline springmintempt_2 & 34985 & 1274 & 28880 & 1016 & 27684 & 880 \\
\hline elev & 34925 & 1334 & 28885 & 1011 & 27673 & 891 \\
\hline springmaxtempt_2 & 34911 & 1348 & 28776 & 1120 & 27659 & 904 \\
\hline nddt_2 & 34662 & 1598 & 28768 & 1128 & 27657 & 907 \\
\hline summermaxtempt_3 & 34661 & 1598 & 28782 & 1115 & 27645 & 919 \\
\hline mintempcoldestt_1 & 34761 & 1498 & 28783 & 1113 & 27623 & 941 \\
\hline summermintempt_2 & 34661 & 1598 & 28780 & 1116 & 27608 & 956 \\
\hline
\end{tabular}


Table S3: Model selection results on defoliation FI transitions also testing submodels of the effect of forest type and years since outbreaks. We removed all data points where the number of years since the last outbreak and the forest type was unknown, which resulted in a smaller data set (192 056 instead of 235744 observations). The three best climate variables were summer maximum temperature $t-3$, summer minimum temperature $t-3$ and number of growing degree days $t-2$. The variables had third-degree response terms. $\mathrm{K}=$ the number of variables.

\begin{tabular}{|c|c|c|c|c|c|c|}
\hline & K & AIC & deltaAIC & pseudo-r $\sim 2$ & random residuals & temporal residuals \\
\hline all climate + dispersal & 79 & 7232 & 0 & 0.300 & 13 & 0 \\
\hline all climate & 76 & 8171 & 939 & 0.234 & 0 & 83 \\
\hline 3 climate + dispersal & 13 & 8764 & 1532 & 0.140 & 75 & 132 \\
\hline 3 climate (with interactions) & 64 & 8861 & 1629 & 0.145 & 53 & 105 \\
\hline dispersal & 4 & 9072 & 1840 & 0.091 & 109 & 153 \\
\hline 1 climate $\mathrm{x}$ dispersal & 16 & 9107 & 1875 & 0.127 & 44 & 136 \\
\hline latitude $x$ longitude & 16 & 9179 & 1947 & 0.111 & 73 & 158 \\
\hline 1 climate + dispersal & 7 & 9183 & 1951 & 0.108 & 68 & 143 \\
\hline 3 climate & 10 & 9469 & 2237 & 0.056 & 100 & 171 \\
\hline global dispersal & 4 & 9721 & 2489 & 0.045 & 93 & 172 \\
\hline years since outbreaks & 4 & 10010 & 2778 & 0.048 & 50 & 176 \\
\hline 1 climate & 4 & 10163 & 2931 & 0.013 & 81 & 185 \\
\hline forest type & 4 & 10238 & 3006 & 0.000 & 87 & 187 \\
\hline 3 climate $\mathrm{x}$ dispersal & 256 & 133008 & 125776 & -6.919 & 935 & 186 \\
\hline
\end{tabular}


Table S4: Model selection results on mortality IF transitions. The predictors had third-degree response terms. $\mathrm{K}=$ the number of variables. The random residuals are estimated on half of the data selected randomly, and repeated 10 times.

\begin{tabular}{l|rrrrr}
\hline & K & AIC & deltaAIC & pseudo-r^2 & rand. res. \\
\hline all climate & 76 & 13700 & 0 & 0.337 & 19 \\
3 climate x dispersal & 256 & 14539 & 839 & 0.314 & 0 \\
latitude x longitude & 16 & 18787 & 5087 & 0.087 & 44 \\
3 climate & 10 & 18230 & 4530 & 0.111 & 48 \\
1 climate & 4 & 18207 & 4507 & 0.104 & 65 \\
\hline
\end{tabular}

\begin{tabular}{l|rrr}
\hline & Forest_K1 & I & Forest_K0 \\
\hline Infected & 7821 & 47368 & 93 \\
Forest & 161975 & 6913 & 65855 \\
\hline
\end{tabular}

Table S5: The total transition events, when t0 is either Infected or Forest with no outbreaks $(K<0.01)$ and with outbreaks $(K>0.01)$.

\begin{tabular}{l|rrr}
\hline & Forest_K1 & I & Forest_K0 \\
\hline Infected & 0.04606 & 0.87264 & 0.00141 \\
Forest & 0.95394 & 0.12736 & 0.99859 \\
\hline
\end{tabular}

Table S6: The total transition probabilities, when $\mathrm{t} 0$ is either Infected, Forest with no outbreaks $(K<0.01)$ and with outbreaks $(K>0.01)$.

\section{References}

Bouchard, M., and I. Auger. 2014. Influence of environmental factors and spatio-temporal covariates during the initial development of a spruce budworm outbreak. Landsc. Ecol. 29:111-126.

Candau, J.-N., and R. A. Fleming. 2011. Forecasting the response of spruce budworm defoliation to climate change in Ontario.

Gouhier, T. C., and A. Grinsted. 2012. biwavelet: Conduct univariate and bivariate wavelet analyses. R package version 0.12.

Gray, D. R. 2013. The influence of forest composition and climate on outbreak characteristics of the spruce budworm in eastern Canada. Can. J. For. Res. 1195:1181-1195.

Régnière, J. 1987. Temperature-dependent development of eggs and larvae of Choristoneura fumiferana (Clem.) (Lepidoptera: Tortricide) and simulation of its seasonal history. Can. Entomol. 119:717-728. 

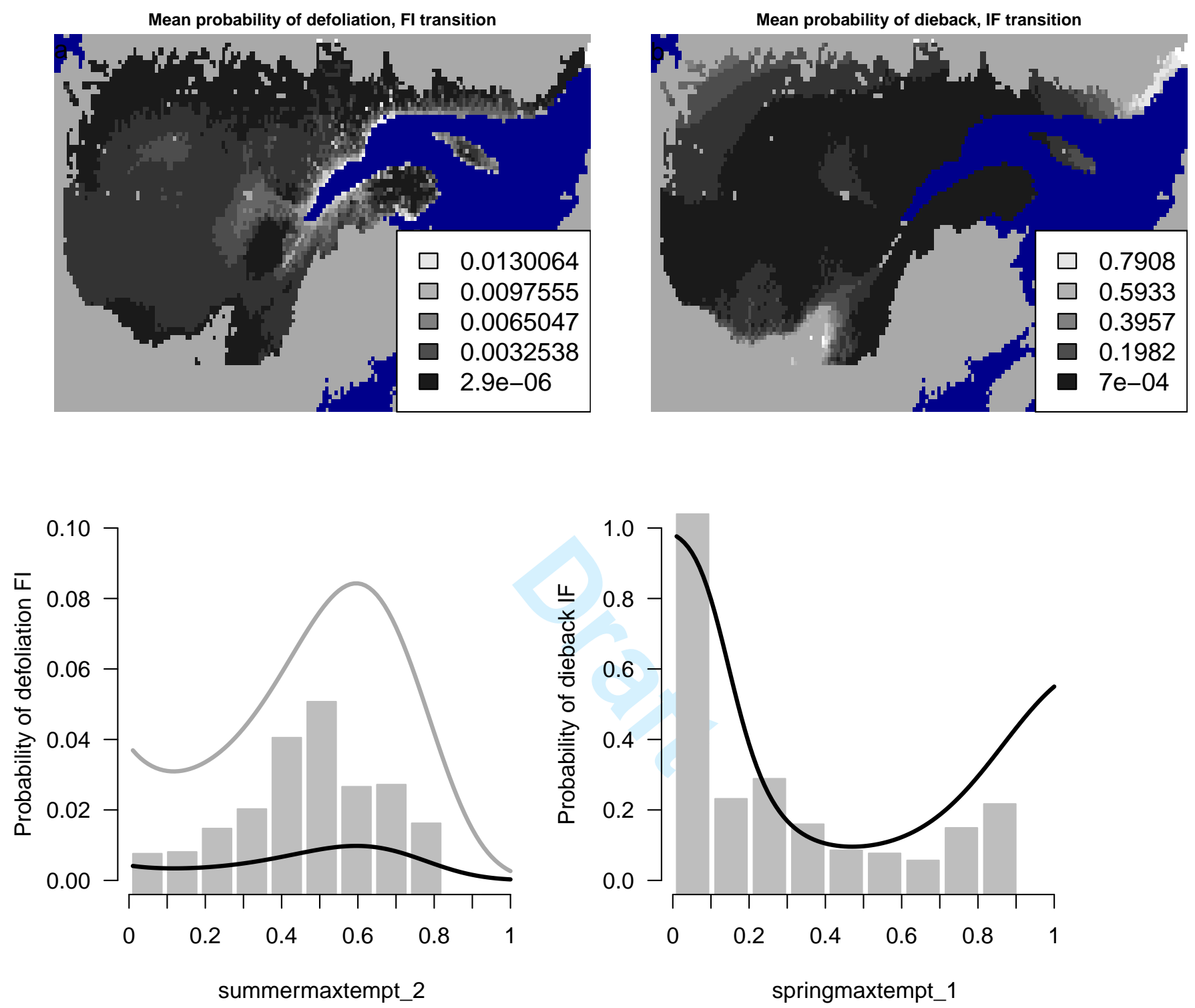

Figure S7: The predicted weather predictions $E$ estimated with a logistic regression with third-degree relationships. Top left, $E_{F I}$, mean probability of forest infection, calculated from all years. White colours indicate a high probability of a forest cell transitioning from the forest state $F$ to defoliated $I$, and black colours indicates a low probability of transition (the forest stays as forest). These probabilities are only based on weather and do not include the effect of defoliation spread. Top right, $E_{I F}$, mean probability of mortality, calculated from all years. White colours indicate a high probability of a forest cell transitioning from the defoliated state $I$ to the forest state $F$, and black colours indicates a low probability of transition (the defoliated area stays defoliated). Bottom left, the effect of one weather variable (selected by backward AIC) on the probability of $F I$ infection. The black line shows the probability of spontaneous infection $\left(K_{i}=0.0\right)$ and the grey line shows the probability of infection when the defoliation spread kernel $K_{i}>0.1$. The grey bars show the observed transitions. Bottom right, the effect of one weather variable (selected by backwards AIC) on the probability of IF mortality. The grey bars show the observed transitions. 


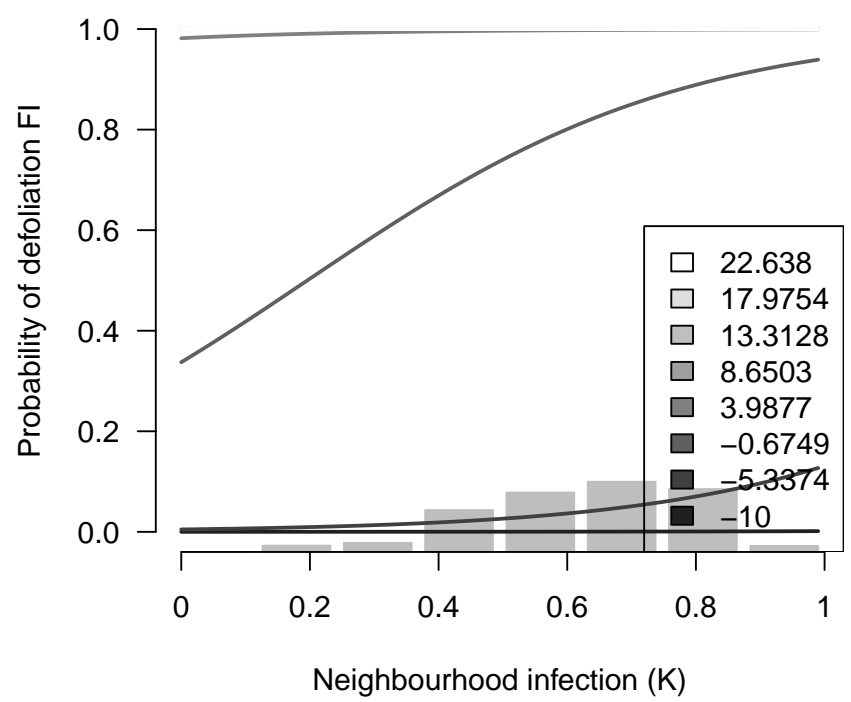

Figure S8: Estimated kernel for the defoliation spread, and the lines represent the different weather predictions.
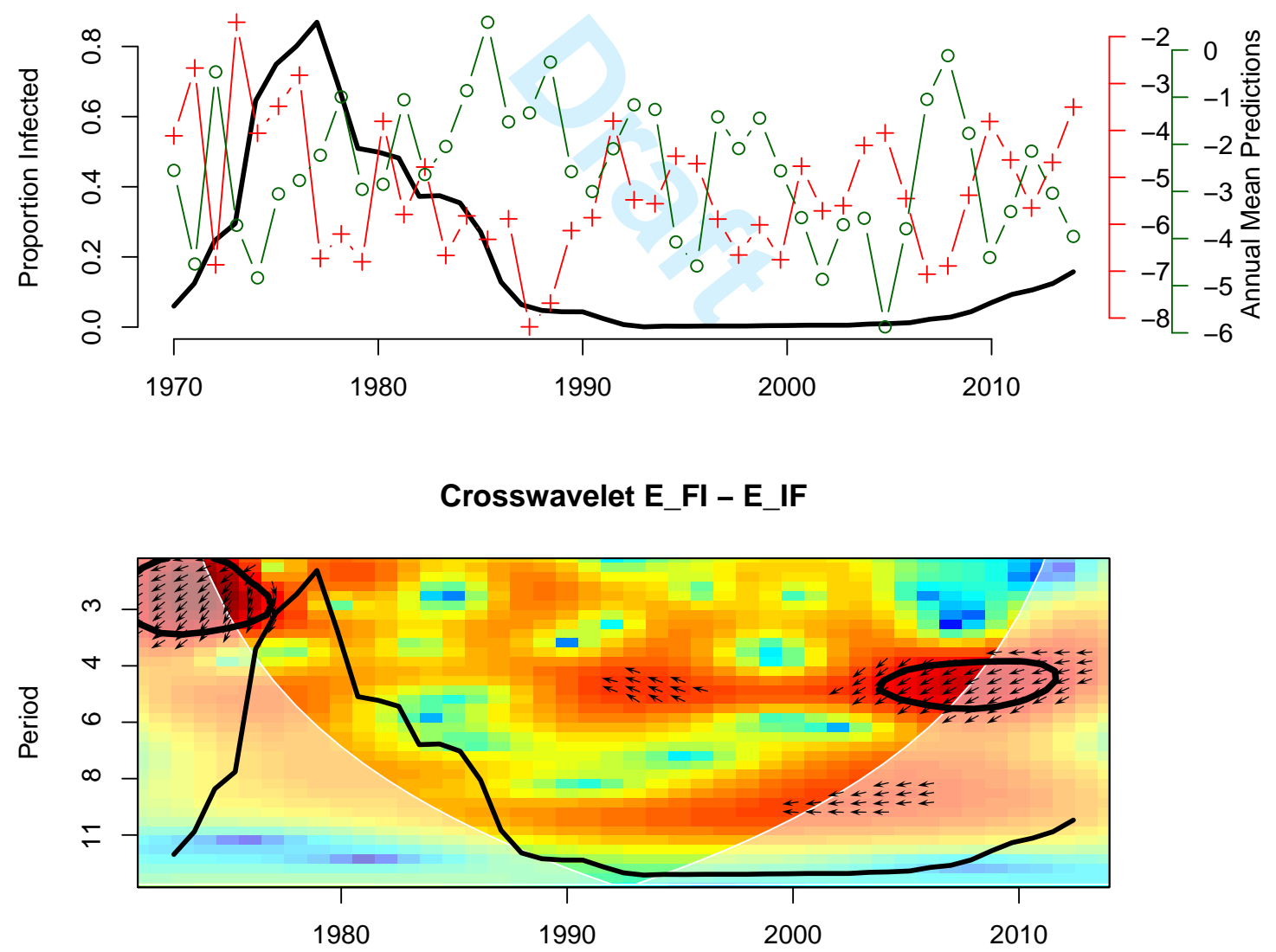

Figure S9: The predicted weather predictions $E$ and defoliation spread parameters. Bottom, the mean predicted probabilities of infection $E_{F I}$ (red lines) and mortality $E_{I F}$ (green lines), in all cells. The solid red line is the annual observed total proportion of defoliated forest. Top, cross-correlation wavelet between $E_{F I}$ and $E_{I F}$. The colours represent the power of the period of the fluctuations, from dark blue (low values), to dark red (high values). The arrows show the phase angles between the fluctuations in the climatic predictions. Arrows pointing left indicate asynchrony in values and arrows pointing down indicate that the $E_{F I}$ fluctuates before the $E_{I F}$ by a quarter period $\left(90^{\circ}\right)$. 